\title{
Exotic Higgs decay via charged Higgs
}

\author{
Tong $\mathbf{L i}^{a}$ and Shufang $\mathbf{S} \mathbf{u}^{b}$ \\ ${ }^{a} A R C$ Centre of Excellence for Particle Physics at the Terascale, \\ School of Physics and Astronomy, Monash University, \\ Melbourne, Victoria 3800, Australia \\ ${ }^{b}$ Department of Physics, University of Arizona, \\ P.O. Box 210081, Tucson, AZ 85721, U.S.A.
}

E-mail: tong.li@monash.edu, shufang@email.arizona.edu

ABSTRACT: The most common search channel for heavy neutral Higgses in models with an extension of the Standard Model Higgs sector is $A / H^{0} \rightarrow \tau \tau$, which becomes ineffective when new decay modes of $A / H^{0}$ open. In this paper, we analyzed two such channels involving charged Higgses in the final states: $A / H^{0} \rightarrow W^{ \pm} H^{\mp}$ and $H^{0} \rightarrow H^{+} H^{-}$. With the consequent decay of $H^{ \pm} \rightarrow \tau \nu$, we found that the limits for $\sigma \times \operatorname{BR}\left(g g \rightarrow A / H^{0} \rightarrow\right.$ $\left.W^{ \pm} H^{\mp}\right) \times \mathrm{BR}\left(H^{ \pm} \rightarrow \tau \nu\right)$ vary from 30 to $10 \mathrm{fb}$ for $m_{A / H^{0}}$ between 300 and $1000 \mathrm{GeV}$ for 95\% C.L. exclusion, and about 80 to $30 \mathrm{fb}$ for $5 \sigma$ discovery. For $H^{+} H^{-}$mode, 95\% C.L. limits on $\sigma \times \mathrm{BR}\left(g g \rightarrow H^{0} \rightarrow H^{+} H^{-}\right) \times \mathrm{BR}^{2}\left(H^{ \pm} \rightarrow \tau \nu\right)$ vary from 9 to $4 \mathrm{fb}$ for $m_{H^{0}}$ between 400 and $1000 \mathrm{GeV}$, while the $5 \sigma$ reach is about 20 to $10 \mathrm{fb}$. We further interpret the cross section limits in the Type II $2 \mathrm{HDM}$ parameter space. While $A \rightarrow W^{ \pm} H^{\mp}$ offers great sensitivity in both $\sin (\beta-\alpha)$ versus $\tan \beta$ and $m_{A}$ versus $\tan \beta$ parameter space, $H^{0} \rightarrow H^{+} H^{-}$can cover most of the parameter space for $H^{0}$. Reach in $H^{0} \rightarrow W^{ \pm} H^{\mp}$ is more limited, especially for $m_{H^{0}}>2 m_{H^{ \pm}}$. It is, however, complementary to $H^{0} \rightarrow H^{+} H^{-}$ when $\operatorname{BR}\left(H^{0} \rightarrow H^{+} H^{-}\right)$is accidentally suppressed.

Keywords: Higgs Physics, Beyond Standard Model

ARXIV EPRINT: 1504.04381 


\section{Contents}

1 Introduction 1

$2 \quad 2 H D M$ and exotic Higgs decay 3

3 Current collider limits 5

4 Collider analyses $\quad 6$

$4.1 g g \rightarrow A / H^{0} \rightarrow W^{ \pm} H^{\mp} \quad 7$

$4.2 \quad g g \rightarrow H^{0} \rightarrow H^{+} H^{-} \quad 9$

5 Implication for the Type II 2HDM 11

$\begin{array}{lll}6 & \text { Conclusion } & 15\end{array}$

\section{Introduction}

The discovery of the SM-like Higgs boson at the LHC is the greatest triumph in particle physics [1-4]. The stabilization of the observed Higgs mass of $126 \mathrm{GeV}$, however, provides strong motivation of physics beyond the Standard Model (SM). In addition, there are puzzles facing particle physics which cannot be explained in the SM, for example, the particle candidate for dark matter and the generation of neutrino mass. Solutions to those problems typically lead to models with an extended Higgs sector. Well known examples include the Minimal Supersymmetric Standard Model (MSSM) [5-7], Next-to-Minimal Supersymmetric Standard Model (NMSSM) [8, 9], and Two Higgs Doublet Models (2HDM) [10-13]. In addition to a SM-like Higgs boson in these models, the low energy spectrum typically includes extra CP-even Higgses, CP-odd Higgses, as well as charged ones.

The discovery of beyond the SM Higgses is an unambiguous evidence for new physics beyond the SM. The search for those extra Higgses, however, is typically challenging. For the extra neutral Higgses at the LHC, most of the current searches focus on the conventional Higgs search channels of $W W, Z Z, \gamma \gamma, \tau \tau$ and $b b$ [14-20]. The production of the extra Higgses is typically suppressed compared to the SM Higgs, either due to their larger masses or their suppressed couplings to the SM particles. The decays of beyond the SM Higgses to the $W W$ and $Z Z$ is absent for the CP-odd Higgs, and could be highly suppressed for the non-SM like CP-even Higgses. The $\tau \tau$ or $b b$ decay modes suffer from either suppressed signal or large SM backgrounds, therefore are only relevant for regions of the parameter space with an enhanced $b b$ or $\tau \tau$ coupling. The search for the charged Higgs is even more difficult. For $m_{H^{ \pm}}>m_{t}$, the cross section for the dominant production channel of $t b H^{ \pm}$ is typically small. The dominant decay mode $H^{ \pm} \rightarrow t b$ is hard to identify given the large $t t$ and $t t b b$ backgrounds [21], while the subdominant decay of $H^{ \pm} \rightarrow \tau \nu$ has suppressed 
branching fraction. In the MSSM, even at the final stage of the LHC running, there is a wedge region in $m_{A}$ versus $\tan \beta$ plane with $\tan \beta \sim 10, m_{A} \gtrsim 300 \mathrm{GeV}$ in which only the SM-like Higgs can be observed at the LHC [22]. Similarly, the reach for beyond the SM Higgses are limited in models with an extended Higgs sector.

In addition to the decays to the SM particles, beyond the SM Higgses can decay via "exotic" modes, i.e., heavier Higgs decays to two light Higgses, or one light Higgs with one SM gauge boson. Examples include ${ }^{1} H \rightarrow A A, H \rightarrow H^{+} H^{-}, H \rightarrow A Z, H \rightarrow W^{ \pm} H^{\mp}$, $A \rightarrow H Z, A \rightarrow W^{ \pm} H^{\mp}$, and $H^{ \pm} \rightarrow A W, H W$ etc. These channels typically dominate once they are kinematically open. The current limits on the searches for beyond the SM Higgses are therefore weakened, given the suppressed decay branching fractions into SM final states. Furthermore, these additional decay modes could provide new search channels for the beyond the SM Higgses, therefore complementary to the conventional search channels.

The study of the exotic Higgs decay modes have caught quite some attention recently. Refs. [23-28] studied $H \rightarrow A Z, A \rightarrow H Z$ decays in bbll, $\tau \tau \ell \ell$, and $Z Z Z$ final states. Refs. $[29,30]$ studied $H^{ \pm} \rightarrow A W, H W$ decay from $t b H^{ \pm}$production for heavier charged Higgs. The study of light charged Higgs produced in top quark decay from $t \bar{t}$ pair production or $t j$ single top production can be found in ref. [31]. Earlier work for Higgs exotic decay can be found in refs. [32-37]. It was found that those exotic Higgs decay modes could be complementary to the conventional Higgs search modes, in particular, in regions of small $\tan \beta$. Thus they offer alternative Higgs search channels in regions where the conventional search channels are ineffective. In particular, searches based on $A \rightarrow Z h^{0}$ and $H^{0} \rightarrow h^{0} h^{0}$ has been performed at the ATLAS and CMS experiments [18, 38, 39].

In this paper, we analyze two other exotic decay modes for the neutral Higgs that involve a light charged Higgs in the final states: $A / H \rightarrow W^{ \pm} H^{\mp}$ and $H \rightarrow H^{+} H^{-}$, with the subsequent decay of $H^{ \pm} \rightarrow \tau \nu$. The SM backgrounds typically involve processes with $W \rightarrow \tau \nu$. Due to the difference in the structure of Yukawa coupling and gauge coupling, and the spin correlation in tau decay, the charged decay product of tau lepton in the signal typically has a harder spectrum comparing to that of the backgrounds, which can be used to separate the signal from the SM backgrounds.

Note that there are strong flavor constraints on the mass of a light charged Higgs [40, 41], in particular, $b \rightarrow s \gamma$ [42]. In addition, precision measurements require the charged Higgs to be nearly degenerate with one of the neutral Higgses [43-48]. Those indirect constraints, however, are typically model dependent and could be relaxed when there are contributions from other sectors in the model [49]. In this paper, we focused on the collider aspect of beyond the SM Higgses and considered the light charged Higgs in the decay chain as long as it satisfies the current direct collider search limits.

The paper is organized as follows. In section 2, we give a brief overview of the $2 \mathrm{HDM}$ and parameter regions where $A / H \rightarrow W^{ \pm} H^{\mp}$ and $H \rightarrow H^{+} H^{-}$can be important. In section 3 , we present the current collider limits on the neutral Higgses as well as the

\footnotetext{
${ }^{1}$ Note that we use $h^{0}$ and $H^{0}$ to refer to the lighter or the heavier CP-even Higgs for models with two CP-even Higgs bosons. When there is no need to specify, we use $H$ and $A$ to refer to the CP-even and CP-odd Higgses respectively.
} 
charged Higgs. In section 4, we present the details of our collider analyses. In section 5, we study the implication of the cross section limits on the parameter space of the Type II 2HDM models. Finally in section 6 we summarize our main results and conclude.

\section{2HDM and exotic Higgs decay}

There are two $\mathrm{SU}(2)_{L}$ Higgs doublets in the $2 \mathrm{HDM}$ :

$$
H_{i}=\left(\begin{array}{c}
h_{i}^{+} \\
\left(v_{i}+h_{i}+i P_{i}\right) / \sqrt{2}
\end{array}\right), i=1,2 .
$$

The neutral component of each Higgs doublet obtains a vacuum expectation value (vev) $v_{1}$ and $v_{2}$ with $v=\sqrt{v_{1}^{2}+v_{2}^{2}}=246 \mathrm{GeV}$ after electroweak symmetry breaking. Three degrees of freedom are eaten by the SM $W$ and $Z$ boson, with the remaining Higgses being two $\mathrm{CP}$ even Higgses $h^{0}$ and $H^{0}$, one $\mathrm{CP}$ odd Higgs $A$ and a pair of charged Higgses $H^{ \pm}$:

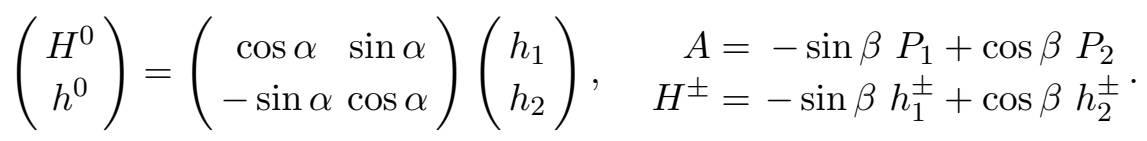

The most general Higgs potential has eight free parameters with the assumption of a discrete $\mathcal{Z}_{2}$ symmetry that can only be softly broken. Two of these parameters are replaced by $v$ and $\tan \beta=v_{2} / v_{1}$, with the remaining five can be chosen as the CP-even Higgs mixing angle $\alpha$, physical Higgs masses $\left(m_{h^{0}}, m_{H^{0}}, m_{A}, m_{H^{ \pm}}\right)$and the $\mathcal{Z}_{2}$ soft breaking parameter $m_{12}^{2}$.

The couplings that are relevant for $H^{0} / A \rightarrow H^{ \pm} W^{\mp}$ are

$$
W^{\mp} H^{ \pm} H^{0}: \frac{g}{2} \sin (\beta-\alpha)\left(p_{H^{ \pm}}-p_{H^{0}}\right)_{\mu}, \quad W^{\mp} H^{ \pm} A: \frac{g}{2}\left(p_{H^{ \pm}}-p_{A}\right)_{\mu},
$$

with $g$ being the SM SU $(2)_{L}$ coupling. While the coupling of the charged Higgs to the heavy CP-even Higgs $H^{0}$ is proportional to $\sin (\beta-\alpha)$, the coupling of the charged Higgs to the CP-odd Higgs $A$ is independent of the mixing angle. Note that if $H^{0}$ is non-SM like with $h^{0}$ being the observed $126 \mathrm{GeV}$ SM-like Higgs, $|\sin (\beta-\alpha)| \sim 1$, which maximizes the $W^{\mp} H^{ \pm} H^{0}$ coupling. Given the strong limits on the light charged Higgs search either from the LEP [50] or from the LHC [19, 20], i.e. $m_{H^{ \pm}}>155 \mathrm{GeV}$, we consider $m_{H^{0}, A}>250 \mathrm{GeV}$ in the $h^{0}-126 \mathrm{GeV}$ case in our analyses.

The above couplings of gauge boson to a pair of Higgses are universal for different types of $2 \mathrm{HDM}$. The Higgs couplings to the fermions, however, is highly model dependent. For the rest of paper, we will work in the framework of the Type II $2 \mathrm{HDM}$, in which $H_{1}$ couples to the down-type quarks and leptons, while $H_{2}$ couples to the up-type quarks. For a review of different types of $2 \mathrm{HDM}$, please see ref. [10].

Figure 1 shows the branching fractions of $A$ as a function of $\tan \beta$. Once $A \rightarrow W^{ \pm} H^{\mp}$ is kinematically open, in general, it quickly dominates over the usual fermionic decay mode $A \rightarrow b b, \tau \tau$ for $\tan \beta \lesssim 20$. For large $\tan \beta$, branching fractions for $A \rightarrow b b, \tau \tau$ increase due to enhanced bottom and tau Yukawa couplings. For small $\tan \beta$, the branching fraction for $A \rightarrow W^{ \pm} H^{\mp}$ decreases slightly since loop induced processes $A \rightarrow \gamma \gamma, Z \gamma$ rise due to 

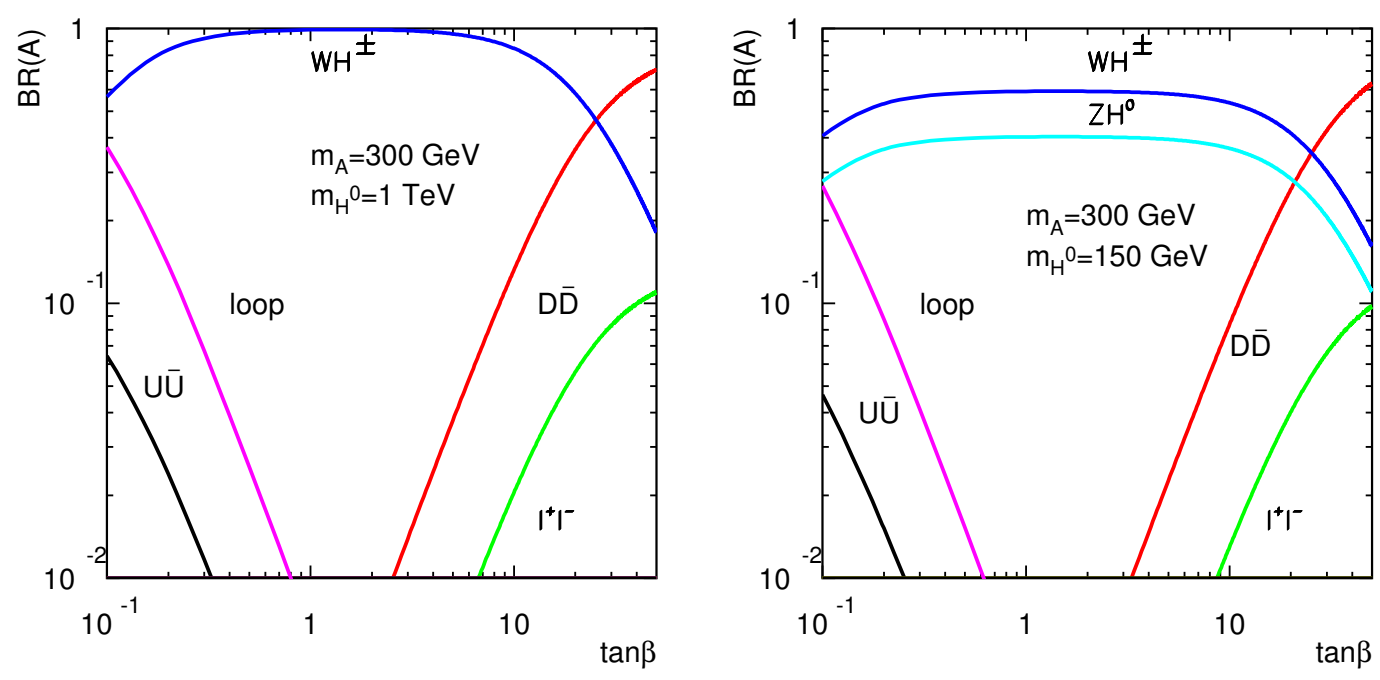

Figure 1. The branching fractions of $A$ as a function of $\tan \beta$ for $m_{A}=300 \mathrm{GeV}, m_{H^{0}}=1 \mathrm{TeV}$ (left panel) or $150 \mathrm{GeV}$ (right panel). We have set $m_{H^{ \pm}}=170 \mathrm{GeV}, m_{h^{0}}=126 \mathrm{GeV}, m_{12}^{2}=0$, and $\sin (\beta-\alpha)=1$. "loop" indicates loop induced Higgs decays of $\gamma \gamma, Z \gamma$ and $g g$.

enhanced top quark contributions. The left panel of figure 1 shows the branching fractions of $A$ when $H^{0}$ decouples. Once $m_{A}>m_{H^{0}}+m_{Z}, A \rightarrow Z H^{0}$ opens and gives a significant contribution in $A$ decay, as shown in the right panel of figure 1 for $m_{H^{0}}=150 \mathrm{GeV}$. $A \rightarrow H^{ \pm} W^{\mp}$, however, still dominates, with branching fraction around $60 \%$ in a large range of $\tan \beta$.

For the heavy CP-even Higgs $H^{0}$, in addition to the decay to $W^{ \pm} H^{\mp}$ as the CP-odd Higgs $A$, it can also decay into a pair of charged Higgs once kinematically open: $H^{0} \rightarrow$ $H^{+} H^{-}$. The relevant coupling receives contributions from all Higgs quartic couplings. In general, it depends on $\tan \beta, \sin (\beta-\alpha), m_{H^{0}}, m_{H^{ \pm}}$as well as $m_{12}^{2}$. Note that there is no $A H^{+} H^{-}$vertex because of the $\mathrm{CP}$ conservation assumption.

In figure 2, the branching fractions of $H^{0}$ are shown as a function of $\tan \beta$ for $m_{H^{0}}=$ $400 \mathrm{GeV}$ (left panel) and $300 \mathrm{GeV}$ (right panel). For $m_{H^{0}}=400 \mathrm{GeV}, H^{0} \rightarrow t \bar{t}$ opens up, which dominates over $H^{0} \rightarrow W^{ \pm} H^{\mp}$ at small $\tan \beta$. Once $H^{0} \rightarrow H^{+} H^{-}$opens, it quickly dominates over other decay modes, unless there is accidental cancelation in $H^{0} H^{+} H^{-}$ couplings around $\tan \beta \sim 1$, as shown by the dip of the brown curve in the left panel of figure 2. With different values of $m_{12}^{2}$, the suppression of $H^{0} \rightarrow H^{+} H^{-}$occurs at different values of $\tan \beta$. When $H^{0} \rightarrow H^{+} H^{-}$is kinematically unaccessible, $H^{0} \rightarrow W^{ \pm} H^{\mp}$ typically dominates over the usual fermionic mode $H^{0} \rightarrow b b, \tau \tau$, except for large $\tan \beta$, as shown in the right panel of figure 2. For $m_{H^{0}}>m_{A}+m_{Z}, H^{0}$ could decay in addition to $A Z$, with decay branching fraction less than that of $H^{0} \rightarrow W^{ \pm} H^{\mp}$. For $m_{H^{0}}>2 m_{A}, H^{0} \rightarrow A A$ opens up, which could compete with $H^{0} \rightarrow H^{+} H^{-}$and $H^{0} \rightarrow W^{ \pm} H^{\mp}$.

Note that in the MSSM, in the decoupling region with $m_{A} \sim m_{H^{0}} \sim m_{H^{ \pm}}, A / H^{0} \rightarrow$ $W^{ \pm} H^{\mp}$ and $H^{0} \rightarrow H^{+} H^{-}$usually do not open due to the limited phase space. These channels, however, could appear either in the MSSM with large loop corrections to the masses or in the NMSSM when extra singlet is introduced [51]. 

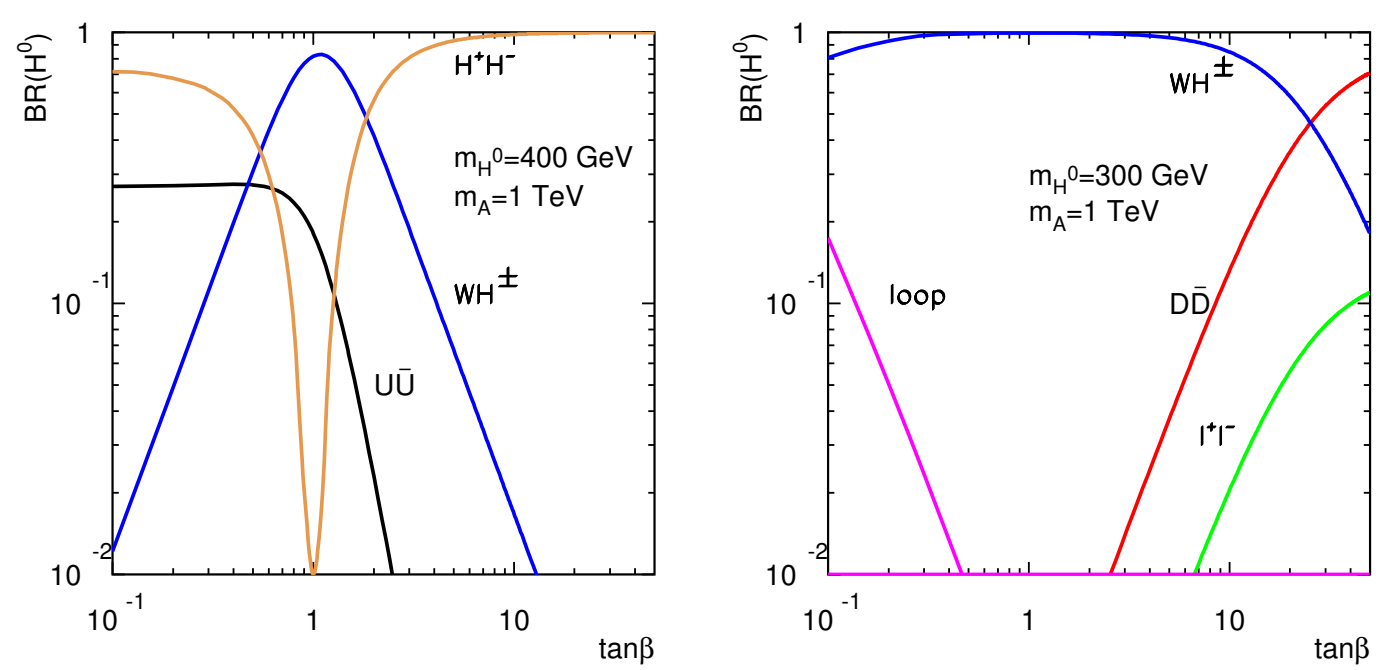

Figure 2. The branching fractions of $H^{0}$ as a function of $\tan \beta$ for $m_{H^{0}}=400$ (left panel) or $300 \mathrm{GeV}$ (right panel). We have set $m_{H^{ \pm}}=170 \mathrm{GeV}, m_{h^{0}}=126 \mathrm{GeV}, m_{A}=1 \mathrm{TeV}, m_{12}^{2}=0$, and $\sin (\beta-\alpha)=1$.

\section{Current collider limits}

The main search mode for the non-SM like neutral Higgses is through $\tau \tau$ channel, which has been performed at both the ATLAS and CMS experiments [14, 15] with $7+8 \mathrm{TeV}$ data set of about $20 \mathrm{fb}^{-1}$ integrated luminosity. For the gluon fusion production, the cross section limits of $g g \rightarrow \phi \rightarrow \tau \tau$ from ATLAS vary between $30 \mathrm{pb}$ to $8 \mathrm{fb}$ for $m_{\phi}$ between 100 to $1000 \mathrm{GeV}$. Interpreting the cross section limits in the MSSM $m_{h}^{\max }$ scenario, a sizable portion of the MSSM parameter space has been ruled out, extending from $\tan \beta \sim 10$ for $m_{A} \sim 100 \mathrm{GeV}$, to $\tan \beta \approx 60$ for $m_{A}=1000 \mathrm{GeV}$ [14]. Limits from CMS are similar [15].

In figure 3, we recast the current 95\% C.L. limit of $p p \rightarrow \phi \rightarrow \tau \tau$ in the $\left(m_{H^{0}}, \tan \beta\right)$ (left panel) and $\left(m_{A}, \tan \beta\right)$ (right panel) planes of the Type II 2HDM. The solid black curves correspond to the limits in the MSSM, when $m_{A} \approx m_{H^{0}}$ with both $A$ and $H^{0}$ contributing to the signal. The solid red curves correspond to the limits in the type II $2 \mathrm{HDM}$, when only contribution from $H^{0}$ or $A$ is included and other non-SM Higgses decouple. The reach is considerably weaker: the current exclusion is about $\tan \beta \sim 12$ at $m_{H^{0}}=200 \mathrm{GeV}$, and $\tan \beta \sim 50$ for $m_{H^{0}}=600 \mathrm{GeV}$, and similar for the CP-odd Higgs $A$. Once $A / H^{0} \rightarrow W^{ \pm} H^{\mp}$ and $H^{0} \rightarrow H^{+} H^{-}$open for $m_{H^{ \pm}}=170 \mathrm{GeV}$, the limits are much more relaxed, as shown by the red dashed curves. In particular, no limit on $m_{H^{0}}$ above $H^{+} H^{-}$threshold can be derived given the strong suppression of $H^{0} \rightarrow \tau \tau$ branching fraction.

Searches with $b b, W W, h^{0} Z$ and $h^{0} h^{0}$ for the non-SM Higgses have also been performed at both the ATLAS and CMS [16-18, 38, 39]. No evidence for a neutral non-SM like Higgs was found and the limits are considerably weaker than the $\tau \tau$ channel.

In our analyses, we consider $A / H^{0} \rightarrow W^{ \pm} H^{\mp}$ and $H^{0} \rightarrow H^{+} H^{-}$, with a benchmark point of $m_{H^{ \pm}}=170 \mathrm{GeV}$. Both ATLAS and CMS have searched for a charged Higgs in $H^{ \pm} \rightarrow \tau \nu, c s$ mode $[19,20]$. For the low mass region of $m_{H^{ \pm}}<m_{t}$, the charged Higgs is produced via top decay, while for the high mass region of $m_{H^{ \pm}}>m_{t}, t b H^{ \pm}$associated 

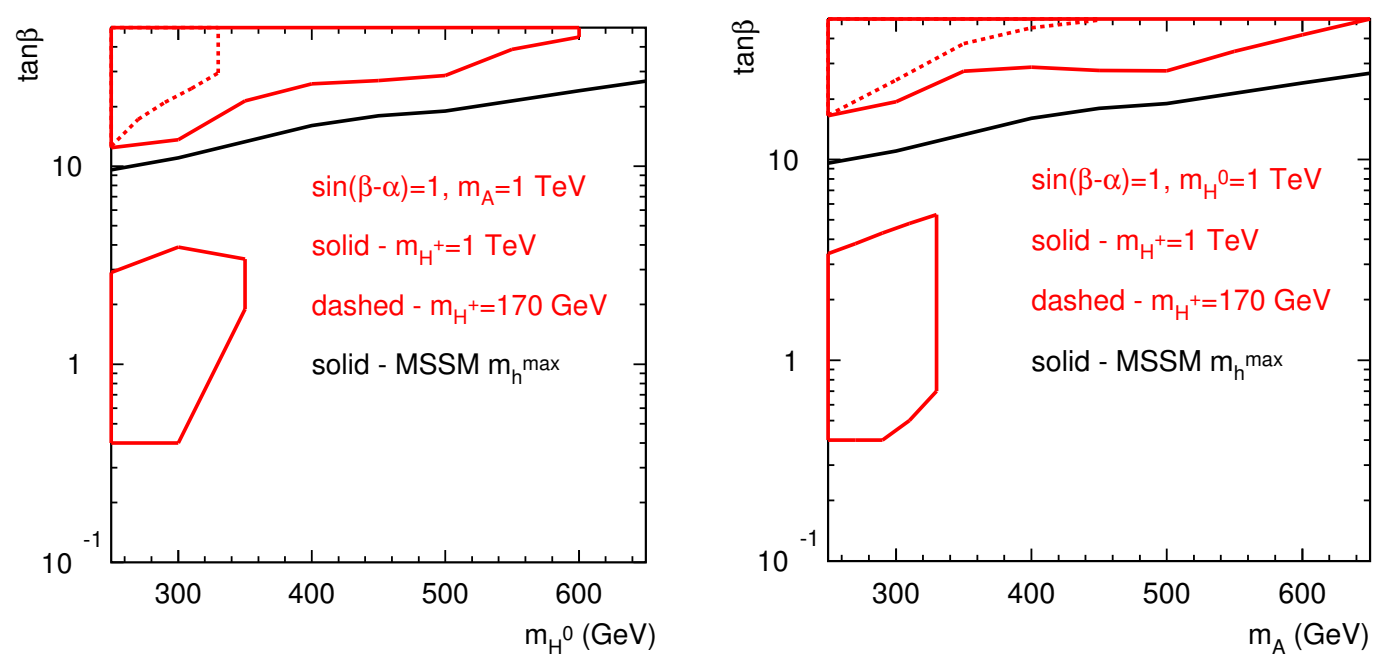

Figure 3. The excluded regions for $H^{0}$ (left) and $A$ (right) in the Type II $2 \mathrm{HDM}$ based on $H^{0} / A \rightarrow \tau \tau$ searches at the $7+8 \mathrm{TeV}$ LHC with $20 \mathrm{fb}^{-1}$ integrated luminosity [14]. The limits for the MSSM $m_{h}^{\max }$ scenario are shown in solid black curves, when both $A$ and $H^{0}$ contribute to the signal. The solid red curves show the limits in the Type II $2 \mathrm{HDM}$ when only $H^{0}$ or $A$ contributes and $m_{H^{ \pm}}=1 \mathrm{TeV}$. The dashed red curves show the relaxed limit for $m_{H^{ \pm}}=170 \mathrm{GeV}$ when $A / H^{0} \rightarrow W^{ \pm} H^{\mp}$ and $H^{0} \rightarrow H^{+} H^{-}$are open. Other parameter choices are $m_{h^{0}}=126 \mathrm{GeV}$ and $\sin (\beta-\alpha)=1$.

production is considered. While most of the low mass region has been excluded for charged Higgs mass less than about $155 \mathrm{GeV}$, there is no limit for $160 \mathrm{GeV}<m_{H^{ \pm}}<m_{t}$ given the suppressed $\mathrm{BR}\left(t \rightarrow b H^{ \pm}\right)$near the threshold. The reach for high mass region only extends down to $m_{H^{ \pm}}>180 \mathrm{GeV}$, due to the overwhelming SM $t \bar{t}$ background at the low mass region.

In addition, there are strong constraints on the non-SM Higgs sector from flavor constraints [40,41] and precision measurements [43-48]. In particular, the latest analyses on $\operatorname{Br}\left(B \rightarrow X_{s} \gamma\right)$ with updated NNLO QCD predictions have constrained the charged Higgs to be heavier than $480 \mathrm{GeV}$ at $95 \%$ C.L. [42]. Precision observables, in particular, $S$ and $T$ oblique parameters, also impose correlations between the charged Higgs mass with the neutral ones: $m_{H^{ \pm}} \sim m_{A}$ or $m_{H^{ \pm}} \sim m_{H^{0}}$. These limits, however, could be relaxed with additional contributions to the flavor or precision observables from other sectors in the new physics models [49]. In this paper, since we focused on the collider aspect of the Higgs exotic decay, we did not apply those constraints explicitly. We chose the mass spectrum of the non-SM Higgses to be characteristic of the exotic decay channels that we analyze. One should, however, keep those potentially dangerous indirect constraints in mind when considering a specific new physics model with an extended Higgs sector.

\section{Collider analyses}

In this section we analyzed $g g \rightarrow A / H^{0} \rightarrow W^{ \pm} H^{\mp}$ and $g g \rightarrow H^{0} \rightarrow H^{+} H^{-}$, with the subsequent decay of $H^{ \pm} \rightarrow \tau \nu$. Due to the spin correlation in $\tau$ decay, the charged product from tau decay is typically harder for the ones from the signal process with tau coming from 
$H^{ \pm}$decay comparing to the ones from the SM backgrounds with $\tau$ mostly from $W$ decay. Both signal and background processes are generated by MadGraph/MadEvent [52] and then passed to TAUOLA to simulate tau lepton decay [53]. We present model independent limits on $\sigma \times \mathrm{BR}$ for both the 95\% C.L. exclusion as well as $5 \sigma$ discovery at the $14 \mathrm{TeV}$ LHC with $300 \mathrm{fb}^{-1}$ integrated luminosity.

\section{$4.1 \quad g g \rightarrow A / H^{0} \rightarrow W^{ \pm} H^{\mp}$}

We studied the gluon fusion production of $A / H^{0}$, followed by $A / H^{0} \rightarrow W^{ \pm} H^{\mp}$ with $H^{ \pm} \rightarrow \tau \nu$ and $W \rightarrow \ell \nu:$

$$
g g \rightarrow A / H^{0} \rightarrow W^{ \pm} H^{\mp} \rightarrow \ell^{ \pm} \tau^{\mp} \nu \bar{\nu}, \quad \ell=e, \mu .
$$

The $\tau$ hadronic decays are adopted to take advantage of spin correlation for the final state hadrons. We consider the two leading hadronic decay modes of tau lepton: $\tau^{ \pm} \rightarrow \pi^{ \pm} \nu_{\tau}$ and $\tau^{ \pm} \rightarrow \rho^{ \pm} \nu_{\tau}$ with $\operatorname{BR}\left(\tau^{ \pm} \rightarrow \pi^{ \pm} \nu_{\tau}\right)=0.11$ and $\operatorname{BR}\left(\tau^{ \pm} \rightarrow \rho^{ \pm} \nu_{\tau}\right)=0.25$. For simplicity, we only display the results of the $\pi$ channel below. The contributions from both $\pi$ and $\rho$ channels are combined for the model independent $\sigma \times$ BR limits.

The leading SM backgrounds are

$$
W^{+} W^{-} \rightarrow \ell^{ \pm} \tau^{\mp} \nu \bar{\nu}, W^{+} W^{-} \rightarrow \tau^{+} \tau^{-} \nu \bar{\nu}, Z Z \rightarrow \tau^{+} \tau^{-} \nu \bar{\nu}
$$

with leading-order $(\mathrm{LO})$ cross section $\sigma_{1}(W W)=3.64 \mathrm{pb}, \sigma_{2}(W W)=0.91 \mathrm{pb}$ and $\sigma(Z Z)=$ $0.3 \mathrm{pb}$, including subsequent $W$ and $Z$ decay. The latter two are followed by one tau decaying leptonic and the other tau decaying hadronically. The reducible backgrounds are

$$
W^{ \pm} Z \rightarrow \ell^{+} \ell^{-} \tau^{ \pm} \nu, \ell^{ \pm} \tau^{+} \tau^{-} \nu
$$

with cross section $\sigma_{1}(W Z)=\sigma_{2}(W Z)=0.21 \mathrm{pb}$ including subsequent decay. These processes have additional $e / \mu$ or $\tau$ lepton and can thus be reduced by vetoing the extra lepton. We apply the $K$-factors of $1.5,1.3$ and 1.7 to the channels $W W, Z Z$ and $W Z$, respectively $[54,55]$.

We select events with one lepton and one hadronically decaying tau satisfying the basic cuts:

$$
p_{T}(\ell) \geq 15 \mathrm{GeV},|\eta(\ell)|<2.5 ; p_{T}\left(h_{\tau}\right) \geq 20 \mathrm{GeV},\left|\eta\left(h_{\tau}\right)\right|<2.3 ; \Delta R_{\ell h_{\tau}} \geq 0.4,
$$

where $h_{\tau}=\pi, \rho$. We veto events with extra leptons or hadronically decaying taus satisfying

$$
\text { veto }: p_{T}(\ell)>7 \mathrm{GeV},|\eta(\ell)|<3.5 ; p_{T}\left(h_{\tau}\right)>10 \mathrm{GeV},\left|\eta\left(h_{\tau}\right)\right|<4.9 \text {. }
$$

To simulate the detector effects, we smear the hadronic/leptonic energy by a Gaussian distribution whose width is parameterized as $[56,57]$

$$
\frac{\Delta E}{E}=\frac{a}{\sqrt{E / \mathrm{GeV}}} \oplus b, \quad a_{\mathrm{had}}=100 \%, b_{\mathrm{had}}=5 \%, a_{\ell}=5 \%, b_{\ell}=0.55 \% .
$$



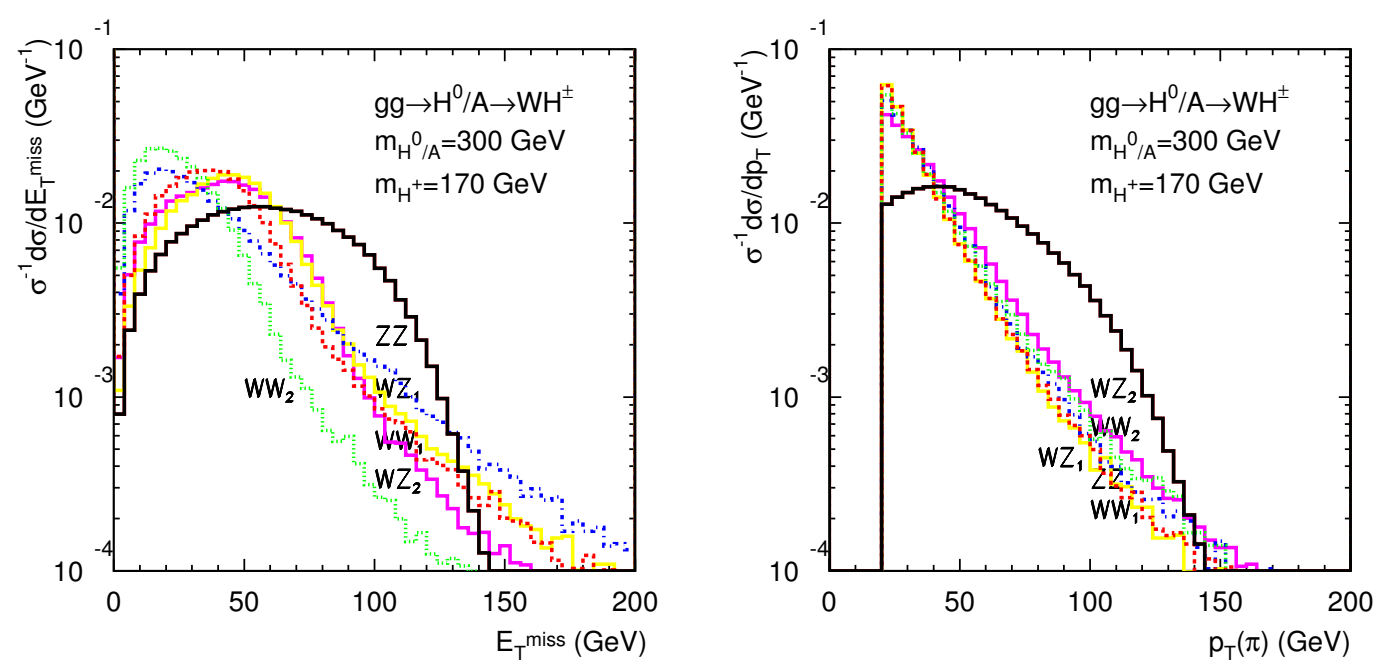

Figure 4. The distributions of $p_{T}(\pi)$ (left panel) and $E_{T}$ (right panel) for signal $W^{\mp} H^{ \pm}$(black) and SM backgrounds after basic cuts in $\tau \rightarrow \pi \nu$ final states. We assume $m_{H^{0} / A}=300 \mathrm{GeV}$ and $m_{H^{ \pm}}=170 \mathrm{GeV}$.

With the above basic cuts and smearing, the distributions of $p_{T}(\pi)$ and $\mathbb{E}_{T}$ for the signal (black curve) and SM backgrounds are shown in figure 4. We note that the signal has a harder $p_{T}(\pi)$ spectrum compared to the backgrounds. This is a well-known result of spin correlation in the $\tau$ decay. For the $H^{+}$signal, the left-handed $\tau^{+}$decays to a right-handed $\bar{\nu}_{\tau}$, causing the $\pi^{+}$to preferentially move along the $\tau^{+}$momentum direction $[58,59]$. In contrast, the $\tau^{+}$coming from a $W^{+}$decay is right-handed which has the opposite effect on the $\pi^{+}$. The distribution for $p_{T}(\rho)$ is similar for $\tau^{ \pm} \rightarrow \rho^{ \pm} \nu$. Signal also has $\mathbb{E}_{T}$ distribution peaked at higher value. We thus tighten the selection cuts by imposing

$$
\mathbb{E}_{T}>50 \mathrm{GeV}, p_{T}(\pi, \rho)>50 \mathrm{GeV} .
$$

We show the $\sigma \times$ BRs of signal and backgrounds before and after cuts, as well as cut efficiencies (with respect to last level of cuts) for $m_{H^{0} / A}=300 \mathrm{GeV}$ and $m_{H^{ \pm}}=170 \mathrm{GeV}$ in table 1 for $\tau^{ \pm} \rightarrow \pi^{ \pm} \nu$. We choose nominal value of $10 \mathrm{fb}$ for the signal cross section. ${ }^{2}$ The dominant background after cuts is the irreducible backgrounds $W W$ with one $W$ decaying leptonically and the other decaying to tau. Utilizing the $\mathbb{E}_{T}$ cut and $p_{T}$ cut, all the backgrounds could be suppressed sufficiently.

The left panel of figure 5 shows the $95 \%$ C.L. exclusion limit (red lines) and $5 \sigma$ discovery reach (black lines) for $\sigma \times \mathrm{BR}\left(g g \rightarrow A / H^{0} \rightarrow W^{\mp} H^{ \pm}\right) \times \mathrm{BR}\left(H^{+} \rightarrow \tau^{+} \nu\right)$ as a function of $m_{H^{0} / A}$ at the $14 \mathrm{TeV}$ LHC with $300 \mathrm{fb}^{-1}$ luminosity, combining both $\pi$ and $\rho$ channels. We have fixed $m_{H^{ \pm}}=170 \mathrm{GeV}$. The solid, dashed and dotted lines are for the limits with no systematic error, $5 \%$ and $10 \%$ systematic errors, respectively. For the neutral Higgs mass between 300 to $1000 \mathrm{GeV}$, the cross section limits vary between 30 to $10 \mathrm{fb}$ for $95 \%$ C.L. exclusion and about 80 to $30 \mathrm{fb}$ for $5 \sigma$ discovery. The cross section limits with $5 \%$ or $10 \%$ systematic error included are about a factor of 7 or 10 worse.

\footnotetext{
${ }^{2}$ We use $\sigma\left(g g \rightarrow A / H^{0}\right)=0.5 \mathrm{pb}, \mathrm{BR}\left(A / H^{0} \rightarrow W^{ \pm} H^{\mp}\right)=100 \%, \mathrm{BR}\left(H^{ \pm} \rightarrow \tau \nu\right)=100 \%$ to get the nominal signal cross section for $\tau \rightarrow \pi \nu$ final states. In general, the signal cross section depends on $\sin (\beta-\alpha)$ and $\tan \beta$ for a given value of $m_{H^{0} / A}$ and $m_{H^{ \pm}}$.
} 


\begin{tabular}{|c|c|c|c|c|c|c|c|c|c|}
\hline Cuts & Signal & $(W W)_{1}$ & $(W W)_{2}$ & $Z Z$ & $(W Z)_{1}$ & $(W Z)_{2}$ & $(W Z)_{2 *}$ & $S / B$ & $S / \sqrt{B}$ \\
\hline$\sigma \times$ BRs $(\mathrm{fb})$ & 10 & 591 & 104 & 30 & 39 & 4.3 & 13.6 & 0.013 & 6.2 \\
\hline basic & 0.67 & 0.13 & 0.068 & 0.11 & 0.0065 & 0.056 & 0.0076 & - & - \\
\hline$E_{T}$ & 0.61 & 0.28 & 0.09 & 0.26 & 0.23 & 0.31 & 0.31 & - & - \\
\hline$p_{T}(\pi)$ & 0.6 & 0.2 & 0.51 & 0.5 & 0.135 & 0.33 & 0.97 & - & - \\
\hline$\sigma \times$ BRs $(\mathrm{fb})$ & 2.45 & 4.2 & 0.33 & 0.43 & 0.0078 & 0.024 & 0.0079 & 0.49 & 19 \\
\hline
\end{tabular}

Table 1. The cross sections (row 2 and 6) and cut efficiencies (row 3-5) of signal $g g \rightarrow A / H^{0} \rightarrow$ $W^{\mp} H^{ \pm} \rightarrow \ell \tau \nu \nu$ and SM backgrounds after various cuts with $\tau^{ \pm} \rightarrow \pi^{ \pm} \nu$ at the $14 \mathrm{TeV}$ LHC. We assume a nominal signal cross section of $10 \mathrm{fb}$. For the background processes, the $K$-factors have been included. $(W Z)_{2}$ and $(W Z)_{2 *}$ refer to $W Z \rightarrow \ell^{ \pm} \tau^{+} \tau^{-} \nu$ with both taus decay to pions for $(W Z)_{2}$ and one tau to pion and one tau to lepton for $(W Z)_{2 *}$. The significance $S / \sqrt{B}$ is given for $300 \mathrm{fb}^{-1}$ luminosity. We assume $m_{H^{0} / A}=300 \mathrm{GeV}$ and $m_{H^{ \pm}}=170 \mathrm{GeV}$.
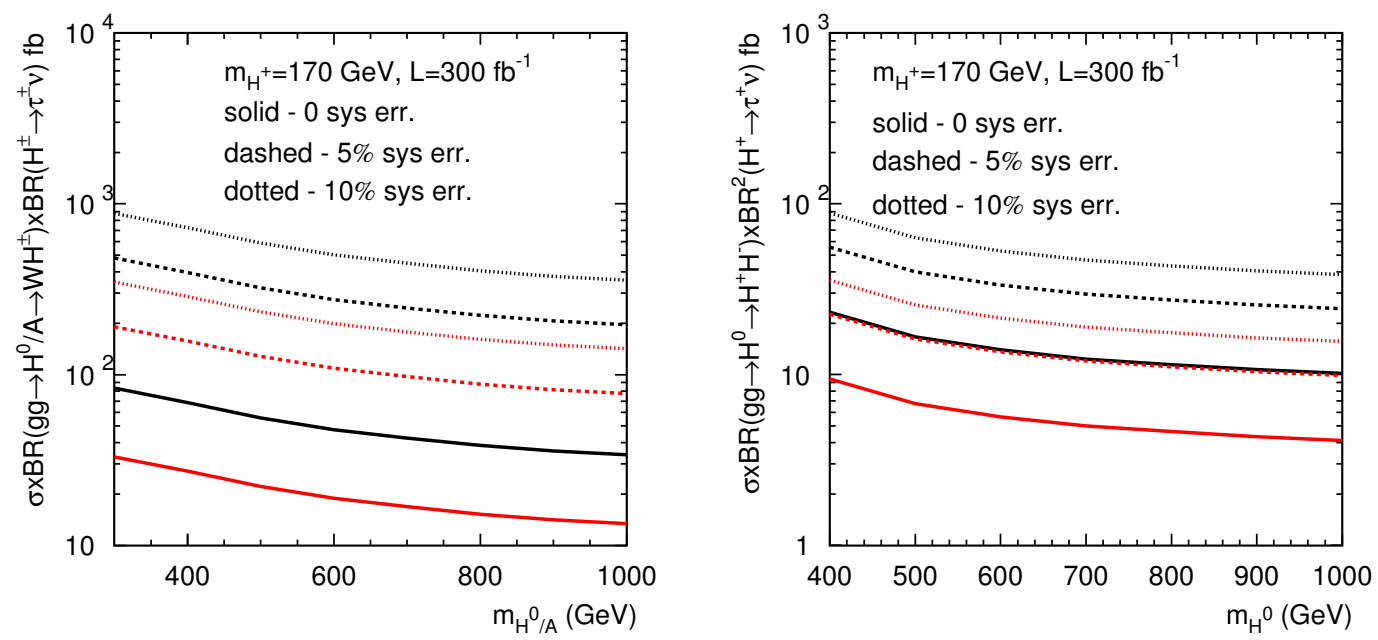

Figure 5. The 95\% CL exclusion limit (red lines) and $5 \sigma$ discovery reach (black lines) of $\sigma \times$ $\mathrm{BR}\left(g g \rightarrow A / H^{0} \rightarrow W^{\mp} H^{ \pm}\right) \times \mathrm{BR}\left(H^{+} \rightarrow \tau^{+} \nu\right)$ vs. $m_{H^{0} / A}$ (left panel) and $\sigma \times \mathrm{BR}\left(g g \rightarrow H^{0} \rightarrow\right.$ $\left.H^{+} H^{-}\right) \times \mathrm{BR}^{2}\left(H^{+} \rightarrow \tau^{+} \nu\right)$ vs. $m_{H^{0}}$ (right panel) at the $14 \mathrm{TeV}$ LHC with $300 \mathrm{fb}^{-1}$ luminosity, with $m_{H^{ \pm}}$fixed at $170 \mathrm{GeV}$. The solid, dashed and dotted lines are for the limits with no systematic error, $5 \%$ and $10 \%$ systematic errors, respectively.

\section{$4.2 \quad g g \rightarrow H^{0} \rightarrow H^{+} H^{-}$}

Another interesting search channel with the charged Higgs in the final states is

$$
g g \rightarrow H^{0} \rightarrow H^{+} H^{-} \rightarrow \tau^{+} \tau^{-} \nu \bar{\nu},
$$

with both taus decaying hadronically. The leading SM backgrounds are

$$
W^{+} W^{-} \rightarrow \tau^{+} \tau^{-} \nu_{\tau} \bar{\nu}_{\tau}, \quad Z Z \rightarrow \tau^{+} \tau^{-} \nu \bar{\nu}, \quad W^{ \pm} Z \rightarrow \ell^{ \pm} \nu_{\ell} \tau^{+} \tau^{-} .
$$

The $W^{ \pm} Z$ background can be reduced by lepton veto.

Adopting the same basic cuts as in eqs. (4.4) and (4.5), the distributions of $E_{T}$ and the invariant mass $m_{\pi \pi}$ for the signal and the SM backgrounds are shown in figure 6 . The 

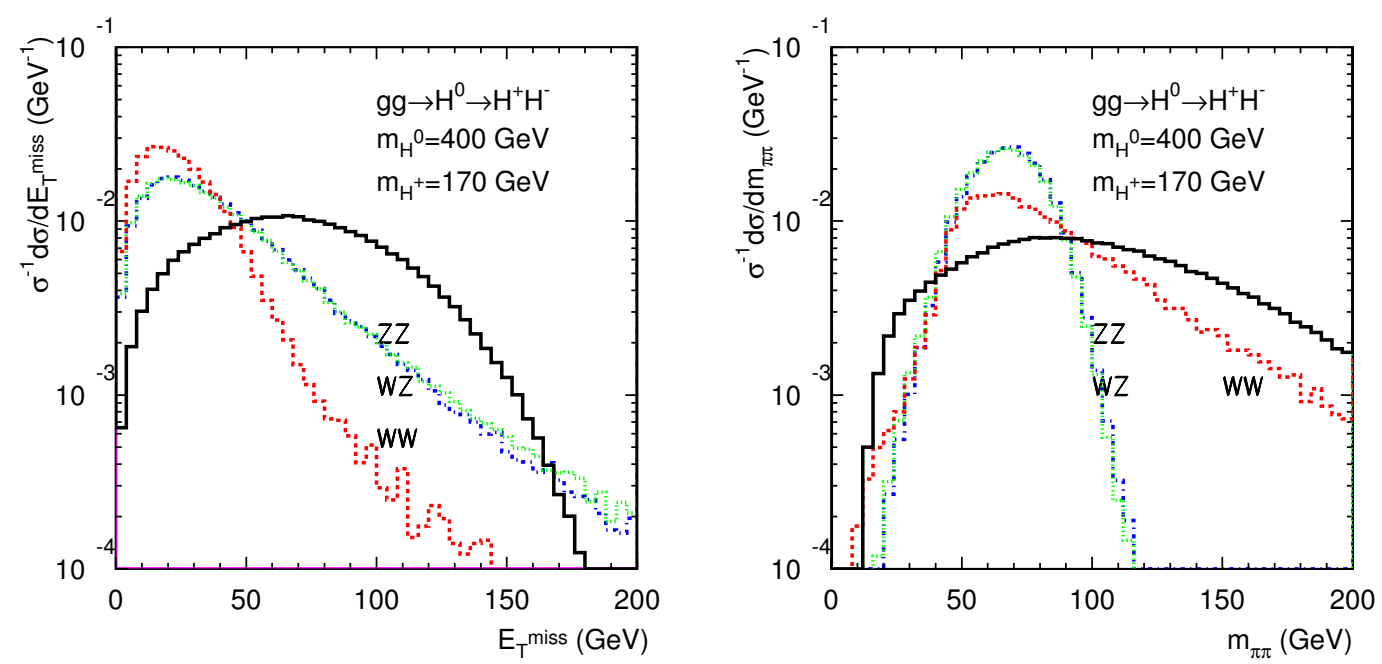

Figure 6. The distributions of $E_{T}$, and $m_{\pi \pi}$ for the signal $H^{+} H^{-}$(black curves) and the SM backgrounds after basic cuts at the $14 \mathrm{TeV}$ LHC in $\tau \rightarrow \pi \nu$ final states. We assume $m_{H^{0}}=400 \mathrm{GeV}$ and $m_{H^{ \pm}}=170 \mathrm{GeV}$.

\begin{tabular}{|c|c|c|c|c|c|c|}
\hline Cuts & Signal & $W W$ & $Z Z$ & $W Z$ & $S / B$ & $S / \sqrt{B}$ \\
\hline$\sigma \times$ BRs $(\mathrm{fb})$ & 1 & 16 & 4.6 & 4.2 & 0.04 & 3.5 \\
\hline basic & 0.62 & 0.04 & 0.14 & 0.01 & - & - \\
\hline$E_{T}$ & 0.7 & 0.1 & 0.33 & 0.2 & - & - \\
\hline$p_{T}^{\max }(\pi)$ & 0.88 & 0.86 & 0.77 & 0.75 & - & - \\
\hline veto $m_{\pi \pi}$ & 0.72 & 0.87 & 0.18 & 0.17 & - & - \\
\hline$\sigma \times \mathrm{BRs}(\mathrm{fb})$ & 0.28 & 0.048 & 0.029 & 0.001 & 3.5 & 17 \\
\hline
\end{tabular}

Table 2. The cross sections and cut efficiencies of the signal $g g \rightarrow H^{0} \rightarrow H^{+} H^{-} \rightarrow \tau^{+} \tau^{-} \nu \bar{\nu}$ and the SM backgrounds after various cuts for $\tau^{ \pm} \rightarrow \pi^{ \pm} \nu$ channel at the $14 \mathrm{TeV}$ LHC. We assume $L=300 \mathrm{fb}^{-1}$ and a nominal cross section for the signal to be $1 \mathrm{fb}$. We fix $m_{H^{0}}=400 \mathrm{GeV}$ and $m_{H^{ \pm}}=170 \mathrm{GeV}$. The $K$-factors for backgrounds are included.

$p_{T}(\pi)$ distribution is very similar to that of figure 4 . Both $\mathbb{E}_{T}$ and $p_{T}(\pi, \rho)$ peak at higher value, similar to the $W^{ \pm} H^{\mp}$ channel. In addition to the $p_{T}(\pi, \rho)$ and $\mathbb{E}_{T}$ cuts the same as in eq. (4.7), we impose $m_{\pi \pi, \rho \rho}$ cuts to reduce the $Z Z$ and $W Z$ backgrounds with $\tau \tau$ pair coming from $Z$ boson:

$$
m_{\pi \pi, \rho \rho}<50 \mathrm{GeV} \text { or } m_{\pi \pi, \rho \rho}>90 \mathrm{GeV} \text {. }
$$

In table 2, we show cut efficiencies as well as the signal and the SM background cross sections before and after cuts, for $g g \rightarrow H^{0} \rightarrow H^{+} H^{-}$with $H^{ \pm} \rightarrow \tau \nu$ for $\tau^{ \pm} \rightarrow \pi^{ \pm} \nu$ final states. The dominant SM background is $W W$, which can be suppressed sufficiently to achieve good signal significance.

In the right panel of figure 5, the $95 \%$ C.L. exclusion limit (red lines) and $5 \sigma$ discovery reach (black lines) for $\sigma \times \mathrm{BR}\left(g g \rightarrow H^{0} \rightarrow H^{+} H^{-}\right) \times \mathrm{BR}^{2}\left(H^{+} \rightarrow \tau^{+} \nu\right)$ are shown as a 


\begin{tabular}{|l|c|c|c|c|}
\hline$\left\{m_{H^{0}}, m_{A}\right\} \mathrm{GeV}$ & $A \rightarrow W^{ \pm} H^{\mp}$ & $H^{0} \rightarrow W^{ \pm} H^{\mp}$ & $H^{0} \rightarrow H^{+} H^{-}$ & $H^{0} \rightarrow A Z$ \\
\hline BP1: $\{1000,300\}$ & $\checkmark$ & - & - & - \\
\hline BP2: $\{300,1000\}$ & - & $\checkmark$ & $\boldsymbol{x}$ & $\boldsymbol{x}$ \\
\hline BP3: $\{400,1000\}$ & - & $\checkmark$ & $\checkmark$ & $\boldsymbol{x}$ \\
\hline
\end{tabular}

Table 3. Benchmark points shown for illustrating the discovery and exclusion limits of $g g \rightarrow$ $A / H^{0} \rightarrow W^{ \pm} H^{\mp}$ and $g g \rightarrow H^{0} \rightarrow H^{+} H^{-}$in the context of the Type II 2HDM. We assume $m_{H^{ \pm}}=170 \mathrm{GeV}$ and $m_{h^{0}}=126 \mathrm{GeV}$. The checkmarks indicate kinematically allowed channels. The "-" means the parent particle being too heavy to be of interesting.

function of $m_{H^{0}}$ at the $14 \mathrm{TeV}$ LHC with $300 \mathrm{fb}^{-1}$ luminosity, combining both $\pi$ and $\rho$ channels. We have fixed $m_{H^{ \pm}}=170 \mathrm{GeV}$. For the neutral Higgs mass between 400 to $1000 \mathrm{GeV}$, the cross section limits vary between 9 to $4 \mathrm{fb}$ for $95 \%$ C.L. exclusion and about 20 to $10 \mathrm{fb}$ for $5 \sigma$ discovery. The cross section limits with $5 \%$ or $10 \%$ systematic error included are about a factor of 2 or 4 worse.

\section{Implication for the Type II $2 \mathrm{HDM}$}

To interpret the $95 \%$ C.L. exclusion and $5 \sigma$ reach limits in the type II $2 \mathrm{HDM}$, we choose three benchmark points as listed in table 3 , with $m_{H^{ \pm}}$fixed to be $170 \mathrm{GeV}$ and $h^{0}$ being the observed $126 \mathrm{GeV}$ SM-like Higgs. BP1 and BP2 with $\left(m_{H^{0}}, m_{A}\right)=(1000,300) \mathrm{GeV}$ or $(300,1000) \mathrm{GeV}$ are the best case scenario for $A \rightarrow W^{ \pm} H^{\mp}$ and $H^{0} \rightarrow W^{ \pm} H^{\mp}$ as other competing decays of $A$ and $H^{0}$ are kinematically unaccessible. BP3 with $\left(m_{H^{0}}, m_{A}\right)=(400$, 1000) $\mathrm{GeV}$ is chosen to illustrate the reach of $H^{0} \rightarrow H^{+} H^{-}$with a suppressed decay branching fraction of $H^{0} \rightarrow W^{ \pm} H^{\mp}$.

The production of $g g \rightarrow A$ only depends on $\tan \beta$ while $g g \rightarrow H^{0}$ depends on both $\tan \beta$ and $\sin (\beta-\alpha)$ [24]. In figure 7, the branching fractions of $A / H^{0} \rightarrow W^{ \pm} H^{\mp}$ are shown for BP1 (left panel) and BP2 (right panel), respectively. The suppression of the $\operatorname{BR}(A \rightarrow$ $\left.W^{ \pm} H^{\mp}\right)$ at $\sin (\beta-\alpha) \sim 0$ is due to the competing $A \rightarrow h^{0} Z$ mode. $\operatorname{BR}\left(A \rightarrow W^{ \pm} H^{\mp}\right)$ gets smaller at both large and small $\tan \beta$ due to the competing $A$ decays to fermions or loop induced processes. For $H^{0} \rightarrow W^{ \pm} H^{\mp}$, the branching fraction is larger than $50 \%$ only for $|\sin (\beta-\alpha)| \gtrsim 0.8$, since $H^{0} W^{ \pm} H^{\mp}$ coupling is proportional to $\sin (\beta-\alpha)$.

In figure 8, the branching fractions of $H^{0} \rightarrow W^{ \pm} H^{\mp}$ and $H^{0} \rightarrow H^{+} H^{-}$are shown in the left and right panel, respectively, for BP3. Comparing to BP2 with $m_{H^{0}}=300 \mathrm{GeV}$, $\mathrm{BR}\left(H^{0} \rightarrow W^{ \pm} H^{\mp}\right)$ receives stronger suppression at both small and large $\tan \beta$ due to the opened $H^{0} \rightarrow H^{+} H^{-}$and $H^{0} \rightarrow t \bar{t}$. It is only significant around a small region near $\tan \beta \sim 1$.

The branching fraction of $H^{0} \rightarrow H^{+} H^{-}$exhibits more complicated dependence on $\sin (\beta-\alpha)$ and $\tan \beta$ due to the $H^{0} H^{+} H^{-}$coupling. The branching fraction is more than $50 \%$ at $\tan \beta \gtrsim 2$ or $\tan \beta \lesssim 0.5$ for $|\sin (\beta-\alpha)|$ close to 1 .

In the left panel of figure 9 , we show the reach in $\sin (\beta-\alpha)$ versus $\tan \beta$ plane for $A \rightarrow W^{ \pm} H^{\mp}$ (BP1). For BP1 with $m_{A}=300 \mathrm{GeV}$, regions above the red (black) curves can be excluded at $95 \%$ C.L. (discovered at $5 \sigma$ ) at the $14 \mathrm{TeV}$ with $300 \mathrm{fb}^{-1}$ luminosity, 

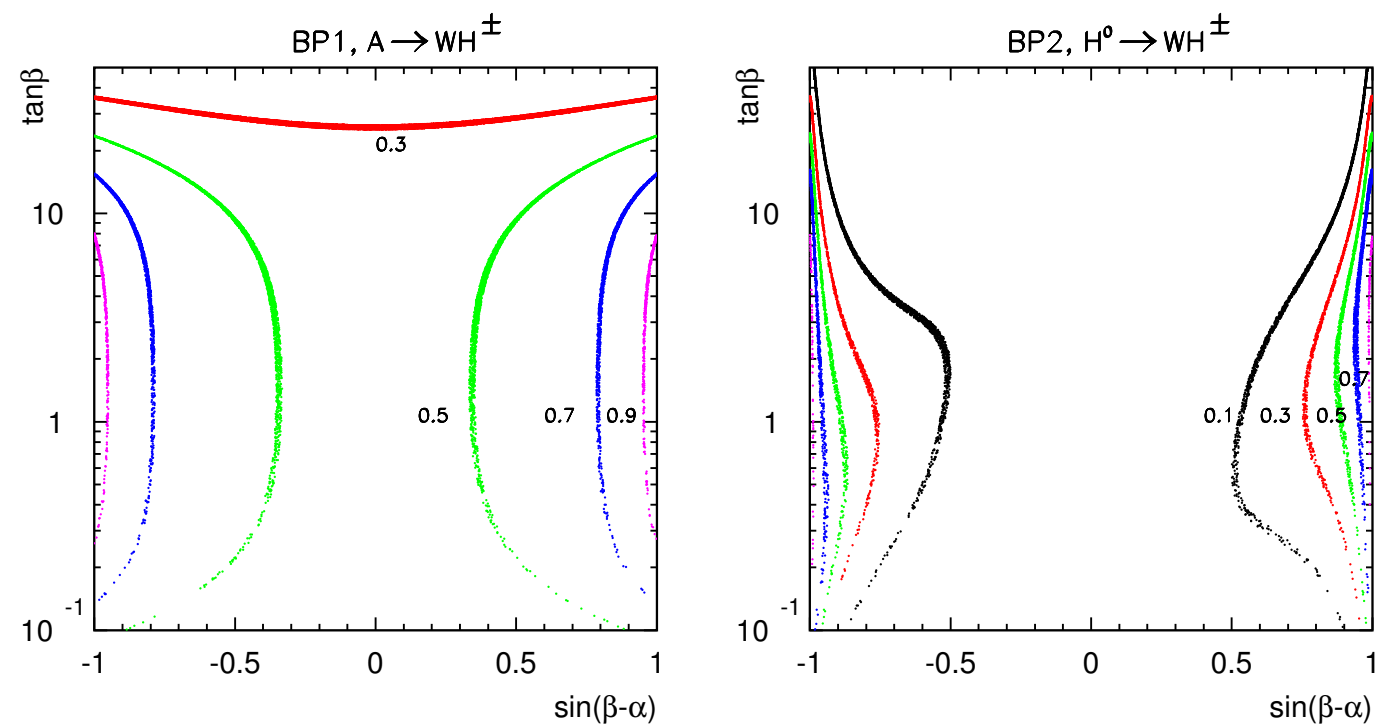

Figure 7. Branching fractions of $A \rightarrow W^{ \pm} H^{\mp}$ for BP1 (left panel) and $H^{0} \rightarrow W^{ \pm} H^{\mp}$ for BP2 (right panel), respectively. We assume $m_{H^{ \pm}}=170 \mathrm{GeV}$ and $m_{h^{0}}=126 \mathrm{GeV}$.
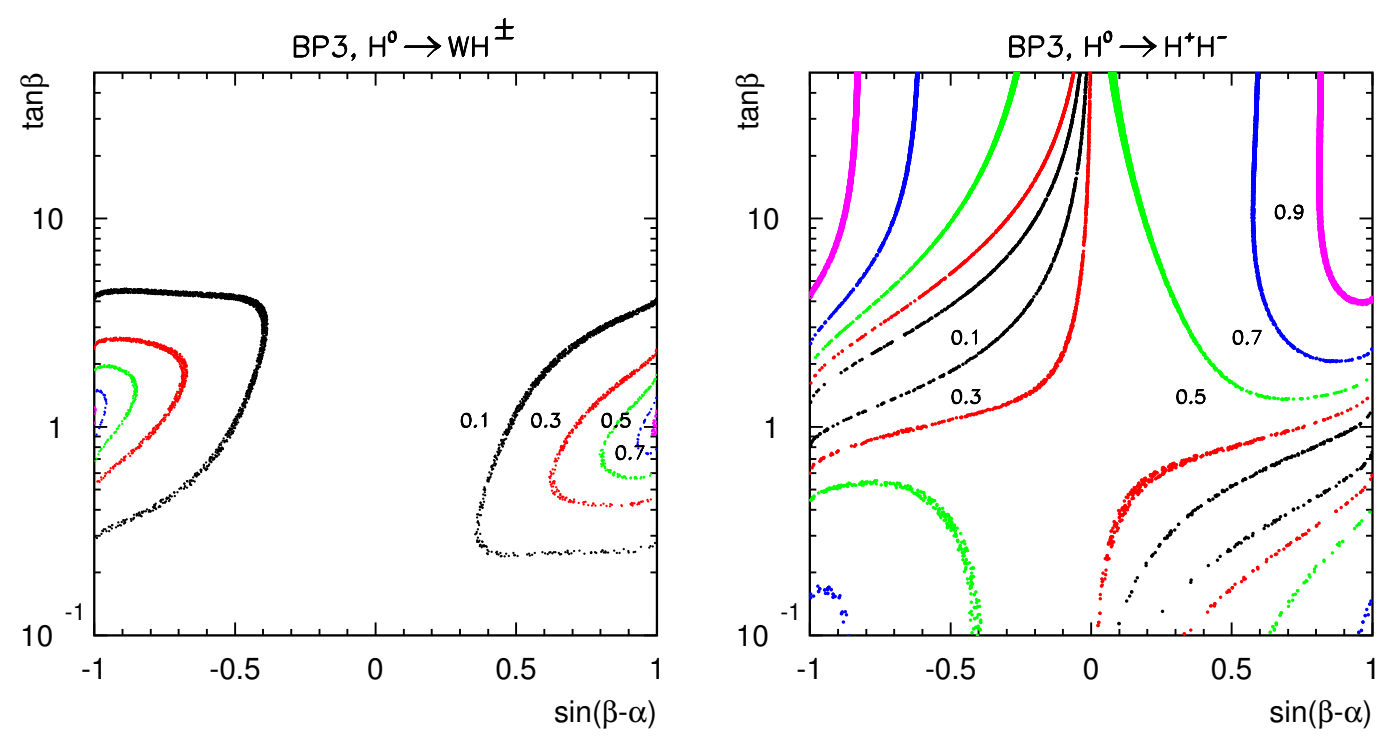

Figure 8. Branching fractions of $H^{0} \rightarrow W^{ \pm} H^{\mp}$ (left panel) and $H^{0} \rightarrow H^{+} H^{-}$for BP3, respectively. We assume $m_{H^{ \pm}}=170 \mathrm{GeV}, m_{h^{0}}=126 \mathrm{GeV}$ and $m_{12}^{2}=0$.

except the region enclosed by the black ellipse around $\tan \beta \sim 10$, which can not be covered by $5 \sigma$ discovery due to the suppression of the production cross section. The region with $\tan \beta \gtrsim 0.2$ can be covered by exclusion for all values of $\sin (\beta-\alpha)$ and $\tan \beta \gtrsim 0.3$ can be covered by discovery except the region with $\tan \beta \sim 10,|\sin (\beta-\alpha)|<0.5$. The loss of sensitivity at small $\tan \beta$ is mainly due to the reduction of $H^{ \pm} \rightarrow \tau \nu$ branching fraction. Reach gets slightly worse for $\sin (\beta-\alpha)$ close to zero due to the competition of $A \rightarrow Z h^{0}$, which suppresses the branching fraction of $A \rightarrow W^{ \pm} H^{\mp}$ correspondingly (see the left panel of figure 7 ). Reach with $10 \%$ systematic error are shown in pink and grey curves 

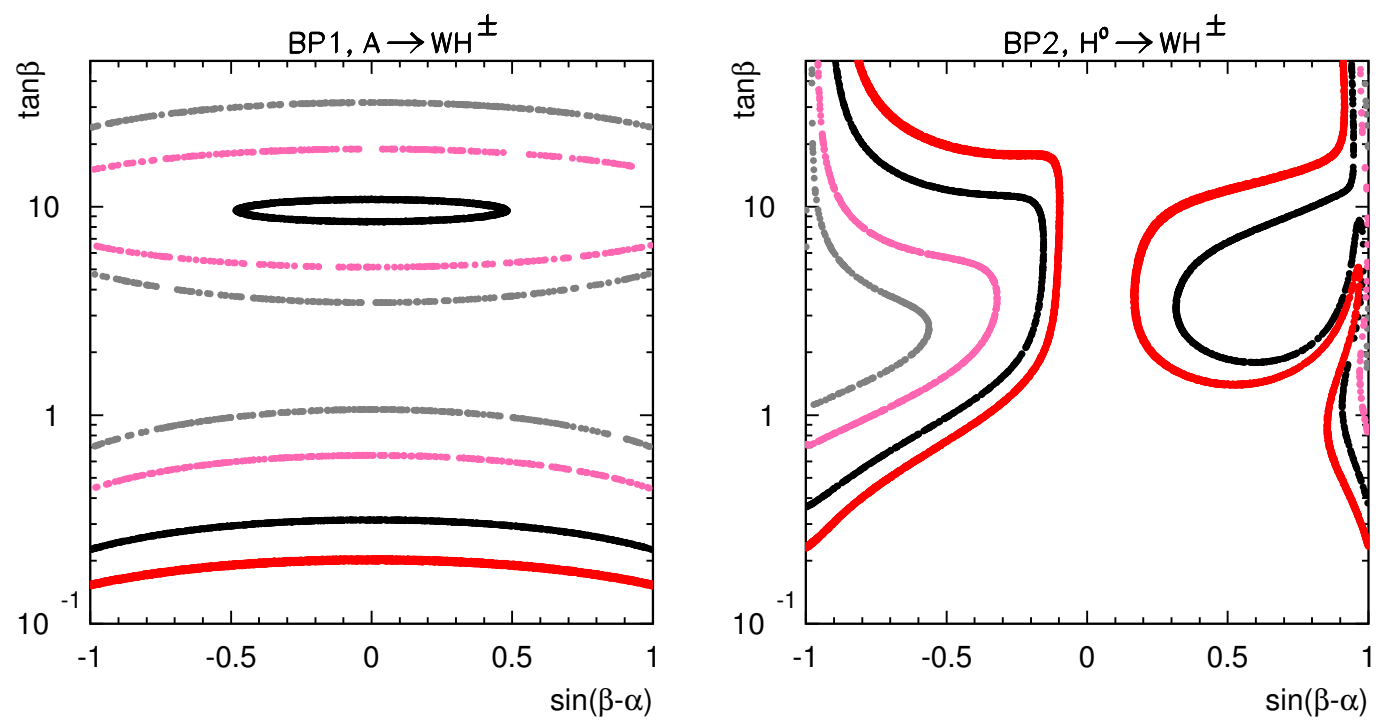

Figure 9. Reach for $g g \rightarrow H^{0} / A \rightarrow W^{\mp} H^{ \pm}$for BP1 (left panel) and BP2 (right panel) at the $14 \mathrm{TeV}$ in $\sin (\beta-\alpha)$ versus $\tan \beta$ plane in the type II $2 \mathrm{HDM}$ at the $14 \mathrm{TeV}$ LHC with $300 \mathrm{fb}^{-1}$ luminosity. The red (pink) and black(grey) lines are 95\% CL exclusion and $5 \sigma$ discovery reach with $0(10 \%)$ systematic error. See text for details.

for $95 \%$ C.L. exclusion and $5 \sigma$ discovery, respectively. The regions shrink considerably comparing to the 0 systematic error case. Only $0.6<\tan \beta<5$ and $\tan \beta>20$ can be excluded and the discovery reach is further reduced to $1<\tan \beta<3$ or $\tan \beta>30$.

In the right panel of figure 9 , we show the reach in $\sin (\beta-\alpha)$ versus $\tan \beta$ plane for $H^{0} \rightarrow W^{ \pm} H^{\mp}$ (BP2), indicated by regions to the left (right) of the curves for negative (positive) $\sin (\beta-\alpha)$. Regions with $\sin (\beta-\alpha)<-0.2$ or $\sin (\beta-\alpha)>0.3$ can be covered by $g g \rightarrow H^{0} \rightarrow W^{ \pm} H^{\mp}$ with $95 \%$ C.L. exclusion. This channel is insensible to region around $\sin (\beta-\alpha) \sim 0$ since $H^{0} \rightarrow W^{ \pm} H^{\mp}$ is highly suppressed. This channel is sensitive to intermediate $\tan \beta$ between 1 to 10, while the reach for $\tan \beta$ is enhanced for $|\sin (\beta-\alpha)| \sim 1$, which is the preferred region for $h^{0}$ being the SM-like $126 \mathrm{GeV}$ Higgs. The decrease in the sensitivity in the thin slice region for $\sin (\beta-\alpha)$ between 0.6 to 0.9 is due to the reduction of $g g \rightarrow H^{0}$ production cross section. The reach for $5 \sigma$ discovery is slightly worse. Introducing $10 \%$ systematic error (regions enclosed by the pink and grey curves) reduces the exclusion and discovery reach further.

The reach of $H^{0} \rightarrow W^{ \pm} H^{\mp}$ for BP3 with $m_{H^{0}}=400 \mathrm{GeV}$ is similar to that of BP2. There is no reach in regions of $\tan \beta \gtrsim 10$ and $\tan \beta \lesssim 0.4$, due to the competing $H^{0} \rightarrow$ $H^{+} H^{-}, t \bar{t}$ modes.

Figure 10 shows the exclusion (region to the left of red/pink curve) and discovery (region to the left of black/grey curve) reach in $m_{A / H^{0}}$ versus $\tan \beta$ plane for $A$ (left panel) and $H^{0}$ (right panel) with $g g \rightarrow A / H^{0} \rightarrow W^{ \pm} H^{\mp}$ channel. For low $m_{A}$ of $300 \mathrm{GeV}, \tan \beta$ as low as 0.2 can be probed, while $\tan \beta>1$ can be excluded for $m_{A}=1000 \mathrm{GeV}$. Reach is reduced for $\tan \beta$ around 10 due to the suppression of the production cross section. Also shown in blue curve is the reduced exclusion by $H^{0} / A \rightarrow \tau^{+} \tau^{-}$[14] due to the opening 

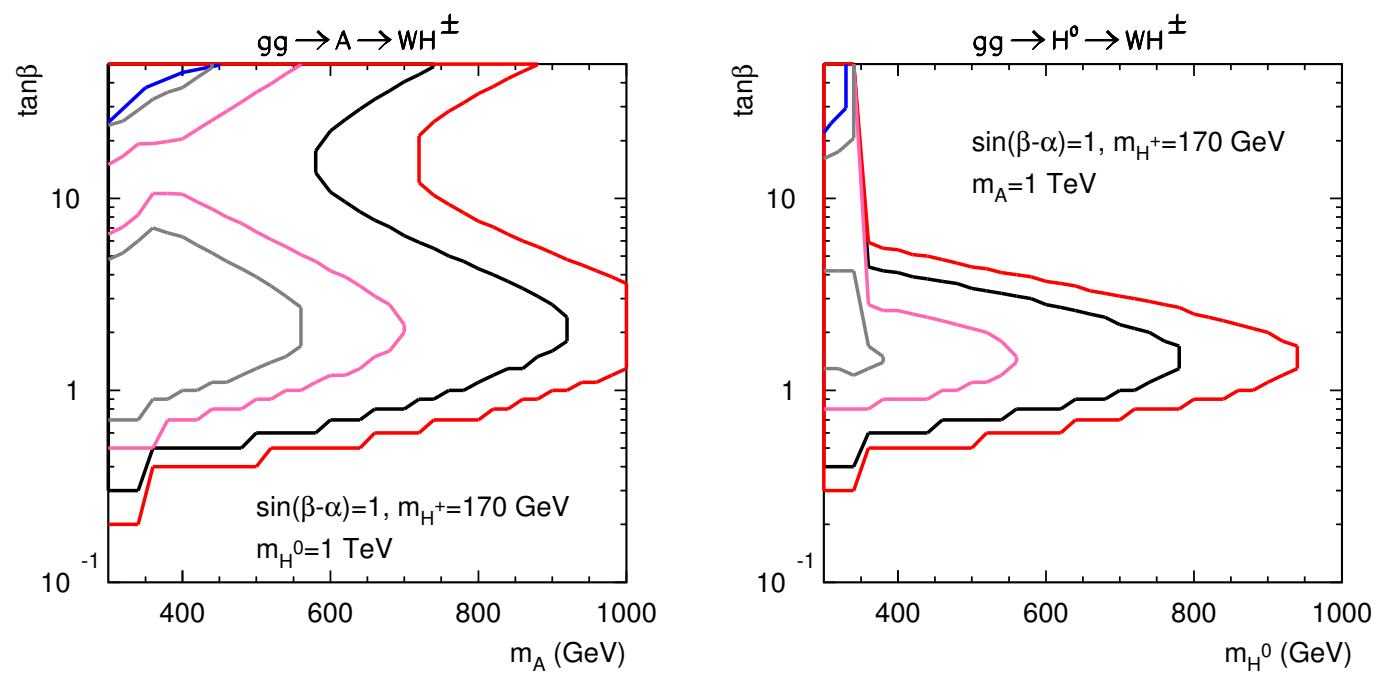

Figure 10. Reach for $g g \rightarrow A / H^{0} \rightarrow W^{ \pm} H^{\mp}$ at the $14 \mathrm{TeV}$ with $300 \mathrm{fb}^{-1}$ luminosity in $m_{A}$ versus $\tan \beta$ plane (left panel), $m_{H^{0}}$ versus $\tan \beta$ plane (right panel) in the type II $2 \mathrm{HDM}$. The blue curve shows the reduced exclusion by $H^{0} / A \rightarrow \tau^{+} \tau^{-}$[14] due to the opening of $A / H^{0} \rightarrow W^{ \pm} H^{\mp}$. Regions to the left of the red/pink (black/grey) curve can be excluded (discovered) assuming 0 and $10 \%$ systematic errors, respectively. We assume $m_{H^{ \pm}}=170 \mathrm{GeV}$ and $\sin (\beta-\alpha)=1$.

of $A \rightarrow W^{ \pm} H^{\mp}$. The reach for the exotic decay of $A \rightarrow W^{ \pm} H^{\mp}$ can cover most of the parameter region in $m_{A}$ versus $\tan \beta$ plane while the conventional search mode of $A \rightarrow \tau \tau$ mode being highly suppressed. For results with $10 \%$ systematic error included (regions to the left of pink/grey curves), the mass reach is about $350 \mathrm{GeV}$ less.

The reach for $H^{0} \rightarrow W^{ \pm} H^{\mp}$ is much more limited, in particular for $m_{H^{0}}>400 \mathrm{GeV}$ since $H^{0} \rightarrow W^{ \pm} H^{\mp}$ is suppressed once $H^{0} \rightarrow H^{+} H^{-}$opens for $m_{H^{0}}>2 m_{H^{ \pm}}$. Regions around $\tan \beta \sim 1$, however, can still be excluded (discovered) for $m_{H^{0}}$ up to 900 (800) $\mathrm{GeV}$ due to the suppression of $H^{0} \rightarrow H^{+} H^{-}$in that region. Given the insensitivity of conventional search channel $H^{0} \rightarrow \tau \tau$ for $\tan \beta \sim 1, H^{0} \rightarrow W^{ \pm} H^{\mp}$ could be an alternative discovery channel for the neutral CP-even Higgs. For results with $10 \%$ systematic error included, the reach is about $400 \mathrm{GeV}$ less.

In the left panel of figure 11, we show the $95 \%$ C.L. exclusion (red/pink) and $5 \sigma$ discovery (black/grey) reach (regions above the curves) in $\sin (\beta-\alpha)$ versus $\tan \beta$ plane for $H^{0} \rightarrow H^{+} H^{-}$for BP3. This channel is sensitive to large region of the parameter space for $\tan \beta>1$. The thin slice of insensitive region at negative $\sin (\beta-\alpha)$ is due to the suppression of $H^{0} \rightarrow H^{+} H^{-}$, while the thin slice of insensitive region at positive $\sin (\beta-\alpha)$ is due to the suppression of $g g \rightarrow H^{0}$.

The right panel of figure 11 shows the reach in $m_{H^{0}}$ versus $\tan \beta$ plane with $g g \rightarrow$ $H^{0} \rightarrow H^{+} H^{-}$channel. Regions with $\tan \beta \gtrsim 1-2$ can be excluded at $95 \%$ C.L. for $m_{H^{0}}$ between 400 to $1000 \mathrm{GeV}$. For results with $10 \%$ systematic error included, the mass reach is about $150 \mathrm{GeV}$ less. The reach around $\tan \beta \sim 10$ is reduced due to the reduction in the production cross section. Combing both $H^{0} \rightarrow W^{ \pm} H^{\mp}$ and $H^{0} \rightarrow H^{+} H^{-}$channels, most regions of $\tan \beta \gtrsim 0.3$ can be covered, while the conventional search channel of $H^{0} \rightarrow \tau \tau$ gets highly suppressed. 

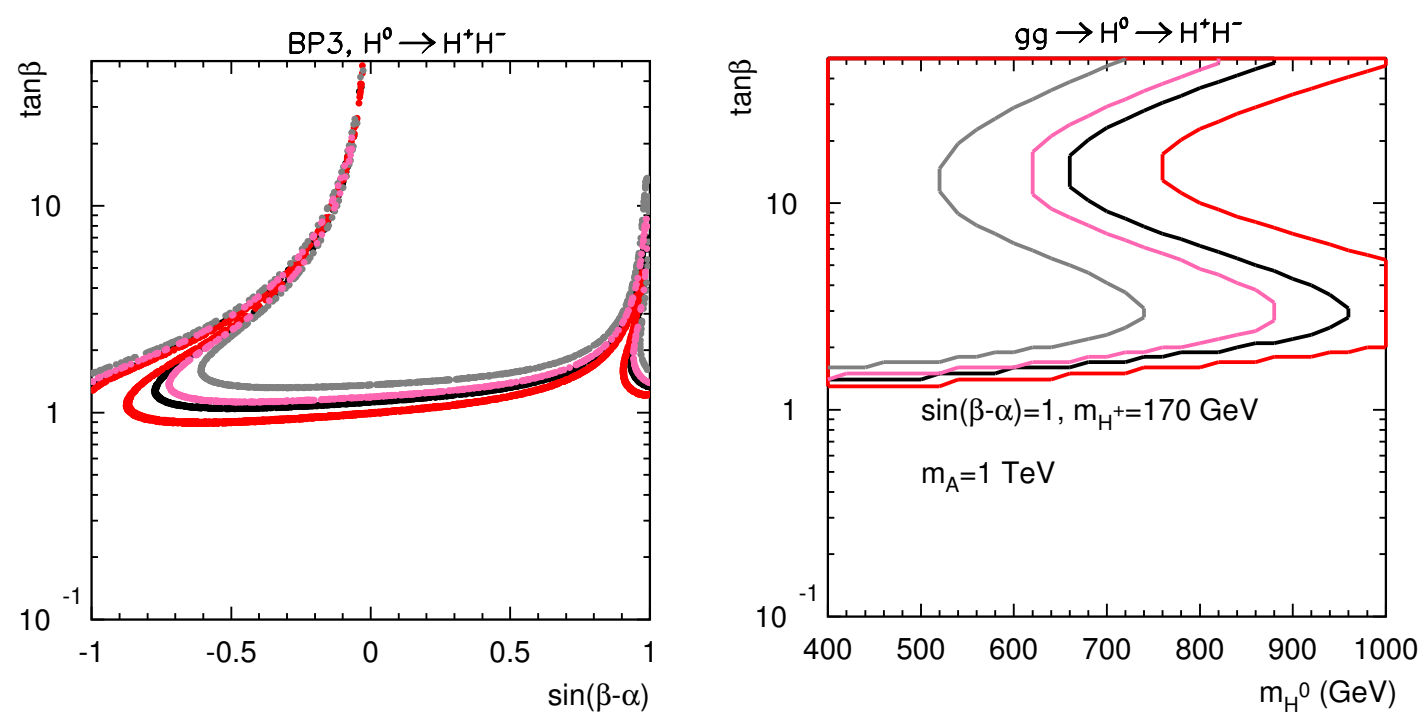

Figure 11. Reach for $g g \rightarrow H^{0} \rightarrow H^{+} H^{-}$at the $14 \mathrm{TeV}$ with $300 \mathrm{fb}^{-1}$ luminosity in $\sin (\beta-\alpha)$ versus $\tan \beta$ plane for BP3 with $m_{H^{0}}=400 \mathrm{GeV}$ (left panel) and $m_{H^{0}}$ versus $\tan \beta$ plane with $\sin (\beta-\alpha)=1$ (right panel) in the type II 2HDM. Regions above (to the left of) the red/pink and black/grey lines are 95\% CL exclusion and $5 \sigma$ discovery reach for the left (right) panel, assuming 0 and $10 \%$ systematic errors, respectively. We assume $m_{H^{ \pm}}=170 \mathrm{GeV}$.

\section{Conclusion}

The conventional search mode for the extra neutral Higgses in models with an extension of the SM Higgs sector is $A / H^{0} \rightarrow \tau \tau$, which is only sensitive to the large $\tan \beta$ region. Furthermore, the opening of the exotic Higgs decay modes, for example $A / H^{0} \rightarrow W^{ \pm} H^{\mp}$ and $H^{0} \rightarrow H^{+} H^{-}$, greatly reduces the sensitivity of the $\tau \tau$ mode. These new decay channels, however, can be used to probe the heavy neutral Higgses, which is discussed in detail in this paper.

One feature of the taus from light $H^{ \pm}$decay is that the decay products of tau, for example, pions and rhos, tend to be more energetic comparing to the SM background taus from $W$ decay. This can be used to suppress the SM backgrounds in both channels that we analysed: $g g \rightarrow A / H^{0} \rightarrow W^{ \pm} H^{\mp} \rightarrow \ell \tau+E_{T}$ and $g g \rightarrow H^{0} \rightarrow H^{+} H^{-} \rightarrow \tau \tau+E_{T}$. For $m_{A / H^{0}}$ between 300 and $1000 \mathrm{GeV}$, we found that the $\sigma \times \mathrm{BR}\left(g g \rightarrow A / H^{0} \rightarrow W^{ \pm} H^{\mp}\right) \times$ $\operatorname{BR}\left(H^{ \pm} \rightarrow \tau \nu\right)$ varies from $30 \mathrm{fb}$ to $10 \mathrm{fb}$ for $95 \%$ exclusion, and about 80 to $30 \mathrm{fb}$ for $5 \sigma$ discovery. For $H^{+} H^{-}$mode, $95 \%$ C.L. limits on $\sigma \times \mathrm{BR}\left(g g \rightarrow H^{0} \rightarrow H^{+} H^{-}\right) \times \mathrm{BR}^{2}\left(H^{+} \rightarrow\right.$ $\tau^{+} \nu$ ) vary from 9 to $4 \mathrm{fb}$ for $m_{H^{0}}$ between 400 and $1000 \mathrm{GeV}$, while the $5 \sigma$ reach is from 20 to $10 \mathrm{fb}$. The cross section limits including $5 \%$ or $10 \%$ systematic error is considerably worse.

We further interpret the cross section limits in the Type II $2 \mathrm{HDM}$ parameter space. For $A \rightarrow W^{ \pm} H^{\mp}$, we found that almost all regions of the parameter space in $\sin (\beta-\alpha)$ versus $\tan \beta$ plane can be covered, except for very small $\tan \beta$ for a benchmark point of $m_{A}=300 \mathrm{GeV}$. It is also sensitive to most regions in $m_{A}$ versus $\tan \beta$ plane with $m_{A}$ up to about $1000 \mathrm{GeV}$, and $\tan \beta$ as low as 1 . It provides an alternative channel to search for the CP-odd Higgs when the conventional mode of $A \rightarrow \tau \tau$ becomes ineffective. 
For the CP-even Higgs $H^{0}$, the most sensitive channel is $H^{0} \rightarrow H^{+} H^{-}$, which covers all regions of the parameter space in $\sin (\beta-\alpha)$ versus $\tan \beta$ plane except for $\tan \beta<1$ for a benchmark point of $m_{H^{0}}=400 \mathrm{GeV}$. In $m_{H^{0}}$ versus $\tan \beta$ plane, $m_{H^{0}}$ up to about $1 \mathrm{TeV}$ can be excluded at $95 \%$ C.L., while $m_{H^{0}}$ up to about $950 \mathrm{GeV}$ can be discovered at $5 \sigma$ significant level. While the reach in $H^{0} \rightarrow W^{ \pm} H^{\mp}$ is more limited, especially for $m_{H^{0}}>2 m_{H^{ \pm}}$, it is complementary to $H^{0} \rightarrow H^{+} H^{-}$mode for regions of $\tan \beta \lesssim 1$. If $10 \%$ systematic error is assumed, the mass reach is typically about $150-400 \mathrm{GeV}$ less.

The discovery of extra Higgses besides the SM-like one would certainly be an unambiguous evidence for new physics beyond the SM. The exotic decay modes of heavy Higgs decaying to two light Higgses, or one Higgs with one SM gauge boson provide alternative search channels, which could greatly enhance the discovery potential for heavy Higgses at current and future colliders. Once those non-SM Higgses are discovered, kinematic reconstruction would provide important information about their mass spectrum. A cross check with the indirect flavor and precision constraints would be complementary and lead to new hints towards new physics beyond the SM.

\section{Acknowledgments}

We thank Felix Kling for helpful discussions on the flavor and precision constraints. The work of T.L. is supported by the ARC Centre of Excellence for Particle Physics at the Terascale. S.S. was supported by the Department of Energy under Grant DE-FG02-13ER41976.

Open Access. This article is distributed under the terms of the Creative Commons Attribution License (CC-BY 4.0), which permits any use, distribution and reproduction in any medium, provided the original author(s) and source are credited.

\section{References}

[1] ATLAS collaboration, Observation of a new particle in the search for the Standard Model Higgs boson with the ATLAS detector at the LHC, Phys. Lett. B 716 (2013) 1 [arXiv:1207.7214] [INSPIRE].

[2] ATLAS collaboration, Combined coupling measurements of the Higgs-like boson with the ATLAS detector using up to $25 \mathrm{fb}^{-1}$ of proton-proton collision data, ATLAS-CONF-2013-034 (2013).

[3] CMS collaboration, Observation of a new boson at a mass of $125 \mathrm{GeV}$ with the CMS experiment at the LHC, Phys. Lett. B 716 (2012) 30 [arXiv:1207.7235] [INSPIRE].

[4] CMS collaboration, Combination of standard model Higgs boson searches and measurements of the properties of the new boson with a mass near $125 \mathrm{GeV}$, CMS-PAS-HIG-13-005.

[5] H.P. Nilles, Supersymmetry, Supergravity and Particle Physics, Phys. Rept. 110 (1984) 1 [INSPIRE].

[6] H.E. Haber and G.L. Kane, The Search for Supersymmetry: Probing Physics Beyond the Standard Model, Phys. Rept. 117 (1985) 75 [INSPIRE].

[7] R. Barbieri, Looking Beyond the Standard Model: The Supersymmetric Option, Riv. Nuovo Cim. 11N4 (1988) 1 [INSPIRE]. 
[8] J.R. Ellis, J.F. Gunion, H.E. Haber, L. Roszkowski and F. Zwirner, Higgs Bosons in a Nonminimal Supersymmetric Model, Phys. Rev. D 39 (1989) 844 [INSPIRE].

[9] M. Drees, Supersymmetric Models with Extended Higgs Sector, Int. J. Mod. Phys. A 4 (1989) 3635 [INSPIRE].

[10] G.C. Branco, P.M. Ferreira, L. Lavoura, M.N. Rebelo, M. Sher and J.P. Silva, Theory and phenomenology of two-Higgs-doublet models, Phys. Rept. 516 (2012) 1 [arXiv:1106.0034] [INSPIRE].

[11] H.E. Haber, G.L. Kane and T. Sterling, The Fermion Mass Scale and Possible Effects of Higgs Bosons on Experimental Observables, Nucl. Phys. B 161 (1979) 493 [InSPIRE].

[12] L.J. Hall and M.B. Wise, Flavor changing Higgs boson couplings, Nucl. Phys. B 187 (1981) 397 [INSPIRE].

[13] J.F. Donoghue and L.F. Li, Properties of Charged Higgs Bosons, Phys. Rev. D 19 (1979) 945 [INSPIRE].

[14] ATLAS collaboration, Search for neutral Higgs bosons of the minimal supersymmetric standard model in pp collisions at $\sqrt{s}=8 \mathrm{TeV}$ with the ATLAS detector, JHEP 11 (2014) 056 [arXiv: 1409.6064] [INSPIRE].

[15] CMS collaboration, Higgs to tau tau (MSSM), CMS-PAS-HIG-13-021.

[16] CMS collaboration, Search for a Higgs boson decaying into a b-quark pair and produced in association with b quarks in proton-proton collisions at $7 \mathrm{TeV}$, Phys. Lett. B 722 (2013) 207 [arXiv: 1302 .2892] [INSPIRE].

[17] ATLAS collaboration, Search for Higgs bosons in Two-Higgs-Doublet models in the $H \rightarrow W W \rightarrow e \nu \mu \nu$ channel with the ATLAS detector, ATLAS-CONF-2013-027 (2013).

[18] CMS collaboration, 2HDM scenario, $H$ to hh and $A$ to Zh, CMS-PAS-HIG-13-025.

[19] ATLAS collaboration, Search for charged Higgs bosons in the $\tau+$ jets final state with $p p$ collision data recorded at $\sqrt{s}=8 \mathrm{TeV}$ with the ATLAS experiment, ATLAS-CONF-2013-090 (2013).

[20] CMS collaboration, Search for charged Higgs bosons with the $H^{+} \rightarrow \tau^{+} \nu_{\tau}$ decay channel in the fully hadronic final state at $\sqrt{s}=8 \mathrm{TeV}$, CMS-PAS-HIG-14-020.

[21] P.S.B. Dev and A. Pilaftsis, Maximally Symmetric Two Higgs Doublet Model with Natural Standard Model Alignment, JHEP 12 (2014) 024 [arXiv:1408.3405] [INSPIRE].

[22] S. Dawson et al., Working Group Report: Higgs Boson, arXiv:1310.8361 [INSPIRE].

[23] B. Coleppa, F. Kling and S. Su, Exotic Higgs Decay via AZ/HZ Channel: a Snowmass Whitepaper, arXiv:1308.6201 [INSPIRE].

[24] B. Coleppa, F. Kling and S. Su, Exotic Decays Of A Heavy Neutral Higgs Through HZ/AZ Channel, JHEP 09 (2014) 161 [arXiv:1404.1922] [INSPIRE].

[25] E. Brownson et al., Heavy Higgs Scalars at Future Hadron Colliders (A Snowmass Whitepaper), arXiv:1308.6334 [INSPIRE].

[26] G.C. Dorsch, S.J. Huber, K. Mimasu and J.M. No, Echoes of the Electroweak Phase Transition: Discovering a second Higgs doublet through $A_{0} \rightarrow Z H_{0}$, Phys. Rev. Lett. 113 (2014) 211802 [arXiv:1405.5537] [INSPIRE]. 
[27] N. Chen, J. Li, Y. Liu and Z. Liu, LHC searches for the CP-odd Higgs by the jet substructure analysis, Phys. Rev. D 91 (2015) 075002 [arXiv:1410.4447] [INSPIRE].

[28] N. Chen, C. Du, Y. Fang and L.-C. Lü, LHC Searches for The Heavy Higgs Boson via Two B Jets plus Diphoton, Phys. Rev. D 89 (2014) 115006 [arXiv:1312.7212] [INSPIRE].

[29] B. Coleppa, F. Kling and S. Su, Charged Higgs search via $A W^{ \pm} / H W^{ \pm}$channel, JHEP 12 (2014) 148 [arXiv: 1408.4119] [InSPIRE].

[30] R. Enberg, W. Klemm, S. Moretti, S. Munir and G. Wouda, Charged Higgs boson in the $W^{ \pm}$ Higgs channel at the Large Hadron Collider, Nucl. Phys. B 893 (2015) 420 [arXiv: 1412.5814] [INSPIRE].

[31] F. Kling, A. Pyarelal and S. Su, Light Charged Higgs Bosons to AW/HW via Top Decay, to appear.

[32] U. Maitra, B. Mukhopadhyaya, S. Nandi, S.K. Rai and A. Shivaji, Searching for an elusive charged Higgs boson at the Large Hadron Collider, Phys. Rev. D 89 (2014) 055024 [arXiv: 1401.1775] [INSPIRE].

[33] L. Basso et al., Probing the charged Higgs boson at the LHC in the CP-violating type-II 2HDM, JHEP 11 (2012) 011 [arXiv:1205.6569] [INSPIRE].

[34] R. Dermisek, J.P. Hall, E. Lunghi and S. Shin, A New Avenue to Charged Higgs Discovery in Multi-Higgs Models, JHEP 04 (2014) 140 [arXiv:1311.7208] [INSPIRE].

[35] B. Mohn, N. Gollub and K.A. Assamagan, The ATLAS discovery potential for a heavy Charged Higgs boson in a large mass splitting MSSM scenario, ATL-PHYS-PUB-2005-017.

[36] K.A. Assamagan, Signature of the charged Higgs decay $H^{ \pm} \rightarrow W h^{0}$ with the ATLAS detector, Acta Phys. Polon. B 31 (2000) 881 [INSPIRE].

[37] K.A. Assamagan, Y. Coadou and A. Deandrea, ATLAS discovery potential for a heavy charged Higgs boson, Eur. Phys. J. direct C 4 (2002) 9 [hep-ph/0203121] [InSPIRE].

[38] ATLAS collaboration, Search for a CP-odd Higgs boson decaying to Zh in pp collisions at $\sqrt{s}=8 \mathrm{TeV}$ with the ATLAS detector, Phys. Lett. B 744 (2015) 163 [arXiv:1502.04478] [INSPIRE].

[39] CMS collaboration, Search for a pseudoscalar boson $A$ decaying into a $Z$ and an $h$ boson in the llbb final state, CMS-PAS-HIG-14-011.

[40] B. Coleppa, F. Kling and S. Su, Constraining Type II 2HDM in Light of LHC Higgs Searches, JHEP 01 (2014) 161 [arXiv:1305.0002] [INSPIRE].

[41] F. Mahmoudi and O. Stal, Flavor constraints on the two-Higgs-doublet model with general Yukawa couplings, Phys. Rev. D 81 (2010) 035016 [arXiv:0907.1791] [InSPIRE].

[42] M. Misiak et al., Updated NNLO QCD predictions for the weak radiative B-meson decays, Phys. Rev. Lett. 114 (2015) 221801 [arXiv: 1503.01789] [INSPIRE].

[43] C.D. Froggatt, R.G. Moorhouse and I.G. Knowles, Leading radiative corrections in two scalar doublet models, Phys. Rev. D 45 (1992) 2471 [inSPIRE].

[44] C.D. Froggatt, R.G. Moorhouse and I.G. Knowles, Two scalar doublet models with softly broken symmetries, Nucl. Phys. B 386 (1992) 63 [INSPIRE].

[45] A. Pomarol and R. Vega, Constraints on CP-violation in the Higgs sector from the $\rho$ parameter, Nucl. Phys. B 413 (1994) 3 [hep-ph/9305272] [INSPIRE]. 
[46] A. Wahab El Kaffas, P. Osland and O.M. Ogreid, Constraining the Two-Higgs-Doublet-Model parameter space, Phys. Rev. D 76 (2007) 095001 [arXiv:0706.2997] [INSPIRE].

[47] H.E. Haber and D. O'Neil, Basis-independent methods for the two-Higgs-doublet model III: The CP-conserving limit, custodial symmetry and the oblique parameters S, T, U, Phys. Rev. D 83 (2011) 055017 [arXiv: 1011.6188] [INSPIRE].

[48] F. Kling, J.M. No and S. Su, Benchmark points for exotic Higgs decays, to appear.

[49] T. Han, T. Li, S. Su and L.-T. Wang, Non-Decoupling MSSM Higgs Sector and Light Superpartners, JHEP 11 (2013) 053 [arXiv: 1306.3229] [INSPIRE].

[50] LEP, DELPHI, OPAL, ALEPH, L3 collaborations, G. Abbiendi et al., Search for Charged Higgs bosons: Combined Results Using LEP Data, Eur. Phys. J. C 73 (2013) 2463 [arXiv: 1301.6065] [INSPIRE].

[51] N.D. Christensen, T. Han, Z. Liu and S. Su, Low-Mass Higgs Bosons in the NMSSM and Their LHC Implications, JHEP 08 (2013) 019 [arXiv: 1303.2113] [INSPIRE].

[52] J. Alwall, M. Herquet, F. Maltoni, O. Mattelaer and T. Stelzer, MadGraph 5: Going Beyond, JHEP 06 (2011) 128 [arXiv: 1106.0522] [INSPIRE].

[53] S. Jadach, Z. Was, R. Decker and J.H. Kuhn, The tau decay library TAUOLA: Version 2.4, Comput. Phys. Commun. 76 (1993) 361 [INSPIRE].

[54] L.J. Dixon, Z. Kunszt and A. Signer, Helicity amplitudes for $O\left(\alpha_{s}\right)$ production of $W^{+} W^{-}$, $W^{ \pm} Z, Z Z, W^{ \pm} \gamma$, or $Z \gamma$ pairs at hadron colliders, Nucl. Phys. B 531 (1998) 3 [hep-ph/9803250] [INSPIRE].

[55] L.J. Dixon, Z. Kunszt and A. Signer, Vector boson pair production in hadronic collisions at order $\alpha_{s}$ : Lepton correlations and anomalous couplings, Phys. Rev. D 60 (1999) 114037 [hep-ph/9907305] [INSPIRE].

[56] CMS collaboration, CMS technical design report, volume II: Physics performance, J. Phys. G 34 (2007) 995 [INSPIRE].

[57] ATLAS collaboration, Expected Performance of the ATLAS Experiment - Detector, Trigger and Physics, arXiv:0901.0512 [INSPIRE].

[58] B.K. Bullock, K. Hagiwara and A.D. Martin, Tau polarization as a signal of charged Higgs bosons, Phys. Rev. Lett. 67 (1991) 3055 [INSPIRE].

[59] B.K. Bullock, K. Hagiwara and A.D. Martin, Tau polarization and its correlations as a probe of new physics, Nucl. Phys. B 395 (1993) 499 [INSPIRE]. 